\title{
Denis Visintin
}

Muzej grada Pazina

Trg Istarskog razvoda 1, HR-52000 Pazin

denis.visintin@inet.hr

\section{L'agro istriano nella seconda metà del XIX secolo}

\author{
Pregledni rad | Review \\ UDK 631(4975-3 Istra)"18"=131.1 \\ $631.17\left(497.5-3\right.$ Istra)" $18^{\prime \prime}=131.1$ \\ Primljeno | Received: 2. VIII. 2014.
}

\section{Sintesi}

A partire dalla metà del XIX secolo, il settore agrario istriano fu interessato da tutta una serie di trasformazioni. Da un lato ciò era dovuto alla politica modernizzatrice che interessò tutta la Monarchia asburgica, dall'altro ci fu la spinta dal basso, ossia dalle autorità provinciali. Nell'articolo si ripercorreranno sommariamente queste vicende, alcune delle quali già trattate dalla storiografia nella bibliografia riportata nelle note, altre invece approfondite dalle ricerche d'archivio dell'autore.

\section{Abstract}

From the mid-19 $9^{\text {th }}$ century, many changes have occurred in Istrian agriculture. On the one hand, they were a consequence of the modernisation policy which was enforced throughout the Austrian Empire; on the other hand they were the result of enticements undertaken by provincial government. This article gives the overview of those events, some of which have already been analysed by historiographers in the cited bibliography, whereas some were more closely analysed in the author's research of the archives.

Parole chiave: agricoltura, economia agricola, storia agraria, sviluppo agrario, crisi agrarie, carestie

Key words: agriculture, agrarian economy, history of agriculture, agricultural development, agricultural crises, shortages 


\section{Introduzione}

Nel corso della seconda metà del XIX secolo, grazie sopratutto all'impegno della Dieta provinciale istriana, iniziò una fase di profondo rinnovamento dell'agricoltura provinciale. Questo processo era dovuto in parte alle novità economiche e sociali avvenute nella prima parte del secolo, e conclusasi con la definitiva abolizione degli oneri feudali nel periodo 1848-1867, a seguito dell'emanazione delle Leggi sull'esonero del suolo. Parzialmente esso era anche una necessità dovuta alle conseguenze disastrose in cui venne a trovarsi a seguito delle crisi e delle epidemie che colpirono il settore agrario, ed a cui l'agricoltura tradizionale non sapeva dare alcuna risposta. Ci fu poi il definitivo processo di ripensamento sul futuro economico ed agrario avviato dalle autorità provinciali. Vi erano poi altre esigenze: la necessità di ridurre i costi produttivi, di lottare efficacemente contro i parassiti e le malattie, di rendere il prodotto altamente concorrenziale, ecc.

Nel testo che segue si ripercorreranno sommariamente queste vicende, alcune delle quali già trattate dalla storiografia nella bibliografia riportata nelle note, altre invece approfondite dalle ricerche d'archivio dell'autore. Manca tuttavia un approccio esaustivo e complessivo che rifletta nel suo insieme questo mezzo secolo di storia agraria regionale.

\section{L'esonero del suolo e l'abolizione del feudalesimo}

Intorno alla metà del secolo la Monarchia fu scossa da una serie di fermenti rivoluzionari, passati alla storia come "Primavera dei popoli", a seguito dei quali, il 26 luglio 1848, la Costituzione viennese abolì tutti i vincoli feudali. La rottura del sistema feudale era ritenuta indispensabile ai fini dell'industrializzazione e della modernizzazione della Monarchia asburgica. Il processo d'abolizione fu comunque molto lungo. Iniziò con le Patenti imperiali del 7 settembre 1848 e del 4 marzo 1849, che decretarono l'abolizione e l'affrancazione degli oneri inerenti al suolo. Successivamente, tra il 1851 ed il 1854 vennero emanate tutta una serie di patenti sovrane che trasformarono tutti coloro che occupavano degli appezzamenti terrieri in proprietari a pieno titolo. I loro effetti furono comunque differenti da luogo a luogo. ${ }^{1}$ Il valore dei terreni fu convertito in denaro, di cui un terzo doveva essere pagato dal vecchio proprietario (entro il 1895), un terzo dal nuovo

1 Gianfranco Battisti, "Di crisi in crisi. Il travaglio di un'economia" (in seguito: "Di crisi in crisi"), Il secolo asburgico 1848 - 1916. Fotografie di un Impero (in seguito: Il secolo asburgico), a cura di Roberto Cassanelli e Zeffiro Ciuffoletti, Trieste 2006, 29. 
proprietario (entro il 1875), un terzo dallo Stato, ovvero dalla provincia, sotto forma di obbligazioni. In parole povere, il costo d'affrancamento della terra dai vincoli feudali fu sopportato dai contribuenti. I titoli d'esonero dovevano essere ammortizzati gradualmente entro quarant'anni, compresi il versamento di capitali ed interessi. ${ }^{2}$

Per portare avanti il lavoro d'esonero, furono istituite delle Commissioni provinciali. I titolari dei diritti estinti furono risarciti in buona parte dai titoli dello stato. Vennero istituiti i Fondi d'esonero del suolo in cui affluivano le somme sopracitate, ed a cui si attingeva per pagare gli interessi sui titoli di riscatto e d'estinzione delle quote annuali di titoli, secondo il piano stabilito. Inizialmente essi erano amministrati dal governo centrale. A partire dal 1862 essi furono sottoposti al controllo delle autorità provinciali. ${ }^{3}$

I precedenti proprietari terrieri feudali si trasformarono in possidenti capitalisti, mentre i contadini, svincolati dai vincoli feudali personali, divennero allo stesso tempo proprietari dei loro piccoli poderi e debitori verso lo stato. Gli effetti della riforma non furono molto utili alla contadinanza, sopratutto a quella delle zone più arretrate della Monarchia, priva di una mentalità monetaria ed imprenditoriale. Nel 1895, poco meno di 4000 proprietari terrieri possedevano ancora oltre il 35\% delle terre della Corona. ${ }^{4}$

Nonostante questi provvedimenti la realtà feudale non cessò del tutto. Alcune categorie continuarono ancora ad esistere. Tali erano ad esempio in Istria i possessi feudali imperiali nei distretti di Pinguente, Parenzo, Montona, Buie, Pirano, Rovigno e Dignano ed i possessi feudali ecclesiastici e quelli privati.

I diritti feudali continuarono ad essere realizzati sotto forma di contributi. Esistevano ancora i notabili, il clero, i popolani ed i contadini, a cui si era affiancato un nuovo ceto, in parte borghese ed in parte nobiliare. Il vecchio ceto notabile dei centri costieri aveva continuato ad esistere ed in alcuni casi si era addirittura rafforzato, aumentando il rispettivo patrimonio.

Il processo d'abolizione feudale riprese con la Legge 12 dicembre 1862, che proibì l'elezione di nuovi feudi, sottolineando ancora una volta

2 Istria nel tempo. Manuale di storia regionale con riferimenti alla città di Fiume (in seguito: Istria nel tempo), a cura di Egidio Ivetic, Collana degli Atti del Centro di ricerche storiche di Rovigno, n. 26, Trieste Rovigno 2006, 456.

3 Almerigo Apollonio, Libertà autonomia nazionalità. Trieste, l'Istria e il Goriziano nell'impero di Francesco Giuseppe: 1848 - 1870 (in seguito: Libertà autonomia nazionalità), Trieste 2007, 125-127.

4. Carlile Aylmer Macartney, L'Impero degli Asburgo 1790-1918, Milano 1981, 519-524; Battisti, "Di crisi in crisi", 30. 
la necessaria abolizione di quelli esistenti, dietro dovuto indennizzo. Nel 1868 anche la Dieta provinciale istriana si schierò decisamente a tale favore. Ad esso si opposero diversi proprietari, intenti a dimostrare che il loro diritto di proprietà aveva un'origine giuridicamente privata, e non feudale. ${ }^{5}$ S’intervenne poi con la Legge del 12 maggio 1869. Essa si riferiva esclusivamente ai vincoli feudali ancora esistenti nelle Contee principesche di Gorizia e Gradisca, nel Margraviato d'Istria e nella città di Trieste, territorio compreso, non contenute nel precedente decreto. ${ }^{6}$

Nel rispetto di essa, l’I. R. Luogotenenza del Litorale istituì l'apposita commissione addetta allo scioglimento dei vincoli feudali, che procedette in merito. Venne inoltre emanato l'apposito Editto per l'insinuazione di tutti i feudi esistenti.

Il lungo perdurare degli oneri feudali, le mancate iniezioni di capitale finanziario, il tradizionalismo e le scarse cognizioni tecnico-pratiche in materia agronomica sia dei possidenti che dei lavoratori agricoli, fecero si che l'obsolescenza agraria durasse a lungo, provocando stagnazione ed arretratezza.

\section{Il rinnovamento agrario}

La fase liberale e di rinnovamento sociale ed economico avviata con l'intento di porre fine alla stasi dell'agro monarchico, compreso quello istriano, impose ai lavoratori delle campagne nuove scelte, ed importanti novità nel settore economico. Iniziò l'era del capitale finanziario e della diffusione dei crediti agrari di cui si servirono i contadini ex dipendenti per procedere all'acquisto della terra svincolata dagli oneri feudali, a tassi d'interesse sfavorevoli. Di conseguenza molti s'indebitarono eccessivamente e dovettero vendere le loro proprietà, ricadendo così ulteriormente in grosse difficoltà economiche. Sostanzialmente però si trattò di tentativi che cozzarono ancora una volta con l'atteggiamento conservativo della popolazione e del ceto proprietario incapace ad avviarsi verso un'economia di tipo monetario e di formazione di capitali per le campagne e ad un uso di tipo razionale del suolo, come sperimentato da Carlo Hugues. ${ }^{7}$

5 Zvane Črnja, "Uloga ezonera u zaoštravanju nacionalnog sukoba u Istri" ("Il ruolo dell'esonero nell'accentuazione del conflitto nazionale in Istria"), Hrvatski narodni preporod u Dalmaciji i Istri, a cura di Jakša Ravlić, Zagabria 1969, 337-338.

6 Archivio regionale di Capodistria, "Fondo Gravisi”, b. 17.

7 Elio Apih, "Appunti sull'agricoltura istriana nell'800”, Atti del Centro di ricerche storiche di Rovigno (in seguito: ACRSR), X, 1979-80, 203-231; Zeffiro Ciuffoletti, "Austria felix. L'Impero austro-ungarico", Il secolo asburgico, 16-17; Carlo Hugues, L'economia agraria dell'Istria settentrionale, Parenzo 1889, 26. 
Gl'interventi innovativi riguardarono anche il settore fiscale. Con la Legge 24 maggio 1869, si procedette all'elaborazione di un nuovo catasto basato sulla misurazione delle singole particelle e sulla determinazione della rispettiva rendita annua desunta dalla loro qualità, nonché classe di coltivazione. Ma anch'esso, come il precedente, portò ben poco sollievo alla padronanza, alla base di un simile modo d'operare rimase il comune censuario. Le operazioni di classificazione e di estimo furono affidate alle commissioni distrettuali, le quali potevano a seconda delle necessità suddividersi ulteriormente in sottocommissioni. Esse istituirono i cosiddetti Fondi modello per ogni genere di coltura e classe. Il loro numero non poteva però superare le otto unità. Dovere delle commissioni era pure di classificare le particelle di confronto. Le tariffe di rendita netta per ogni genere di coltura e classe vennero compilate dalla commissione provinciale. Spettava invece alla commissione centrale la revisione ed il coordinamento degli elaborati delle commissioni provinciali. Seguirono anni di lavoro, cui seguirono le rimostranze, che si potevano presentare nel periodo 1 novembre $1880-30$ maggio 1881.

Il processo innovativo non fu sempre appoggiato e seguito dalle autorità centrali e locali, ed anche la padronanza si dimostrò spesso passiva in merito. Nonostante i miglioramenti avviati, il settore agrario rimase scarsamente competitivo, imparagonabile con quello europeo occidentale, mostrando ancora agli inizi del XX secolo, nelle relazioni degli esperti, delle insufficienze infrastrutturali e paesaggi in degrado. Una delle cause andava probabilmente cercata nell'incapacità di agganciarsi a quelle forme innovative di sviluppo che le vicine aree altoadriatiche avevano effettuato con successo rivolgendosi all'industrializzazione. ${ }^{8}$

L'Occidente europeo aveva da tempo avviato una politica economica d'aumento della produzione alimentare senza una corrispondente crescita delle braccia da lavoro agricolo, consentendo un'indisturbata crescita della popolazione urbana e l'allargamento dei beni e dei servizi cittadini. L'agricoltura tradizionale lasciò il passo a quella industriale. Fu abbandonata la coltivazione di sussistenza e la sola produzione dei generi di primo consumo, ed il sistema tradizionale delle rotazioni agrarie a maggese. S'introdussero nuove coltivazioni di largo consumo, in precedenza sos-

8 Giulio Mellinato, "L'estremità periferica. Una prospettiva economica dell'Istria (1891-1943)", in Livio Dorigo - Giulio Mellinato - Biagio Mannino, Istria Europa. Economia e Storia di una regione periferica, Trieste 2012, 24-27. 
pettate da pregiudizi: la patata e il mais, che levarono il continente dalla fame. Iniziò l'era delle sementi selezionate e dell'aratro metallico. Il maggese fu sostituito con l'introduzione di colture fruttuose nelle rotazioni. Aumentò il numero di animali grazie alla maggiore disponibilità di foraggio. Questa aumentata produttività favorì un maggior interscambio tra città e campagna, la crescita demografica, e migliori vie di comunicazione. Sparivano i beni collettivi, la figura del contadino tradizionale fu sostituita da quella dei proprietari - imprenditori terrieri e dai salariati. La meccanica e la chimica si misero lentamente al servizio dell'agricoltura. Il feudalesimo lasciava spazio ad un nuovo tipo di società. Se ancora negli ultimi decenni del Settecento l'agricoltura era la fonte principale di occupazione e di ricchezza per il 70-80\% della popolazione europea occidentale, un secolo dopo la situazione era molto cambiata. Lagricoltura, che fino ad allora aveva trainato l'industrializzazione, si trovò a sua volta ad essere scavalcata da quest'ultima. Fu per questo che l'ultima grande carestia europea, quella irlandese degli anni 1845-51 non fece grossi danni, ed i mali successivi fecero sì danni ma non compromisero la sussistenza della popolazione. Permaneva però nelle campagne la coabitazione con l'animalia nelle case, l'ammasso familiare in un unico locale e l'assenza di aerazione, di camini senza tiraggio. ${ }^{9}$

Si diffusero anche i concimi chimici, il cui uso rivoluzionò le pratiche agrarie e segnò l'inizio dell'intervento industriale nel settore, favorì l'abbandono del concime umano e dello stallatico animale. ${ }^{10}$

La penisola istriana indicava invece una situazione alquanto diversa ed uno sviluppo ritardato rispetto a quello europeo occidentale, similmente a quanto avveniva nella Monarchia, fin quando questa non promosse il suo processo d'ammodernamento. Dopo le crisi dei primi due decenni del secolo, l'agro peninsulare rinacque e si riprese lentamente, continuando a mostrare un carattere prettamente agricolo di sussistenza. All'incirca tre quarti della popolazione dipendeva dalle risorse offerte da un'agricoltura ancora troppo dipendente dagli eventi climatici, ed in cui predominavano i proprietari terrieri che, unitamente ai braccianti, ai coloni ed ai lavoratori salariati, costituivano i tre quarti delle forze produttive distanziando larga-

9 Roger-Henri Guerrand, "Spazi privati”, La vita privata. L'Ottocento, a cura di Philippe Ariès - Georges Duby, Bari 2001, 279; Paolo Macry, Introduzione alla storia della società moderna e contemporanea, Bologna 1983, 121-152; Hagen Schulze, Aquile e leoni. Stato e nazione in Europa, Bari 1995, 247-250; Bernard Hendrik Slicher van Bath, Storia agraria dell'Europa occidentale (500 - 1850), Torino 1972, 332-449.

10 Guerrand, "Spazi privati”, 266. 
mente i marittimi ed i pescatori, gli artigiani ed i manifatturieri, nonché gli altri professionisti e salariati. La situazione istriana di allora rispecchiava quella europea occidentale del secolo precedente, con qualche novità. Il primo Ottocento istriano fu contrassegnato dall'introduzione della patata e del granoturco, che mutarono le condizioni alimentari. Cambiò anche il paesaggio agrario: la coltura olearia si era ristretta a poche zone, aumentarono gli arativi ed i vigneti, la produzione dei bozzoli di seta e dell'avena diminuivano. Il patrimonio zootecnico era in aumento. Questi piccoli segni di progresso a lungo andare non evitarono però crisi e carestie alla penisola. I centri costieri e quelli urbani dell'entroterra erano il punto di riferimento della vita economica e di mercato, e sede abitativa dei proprietari terrieri. La costa poteva fare affidamento all'attività di piccolo cabotaggio e di pesca. La marineria era concentrata a Trieste. Una certa industrializzazione la si ebbe nell'ambito cantieristico ed in alcuni settori produttivi, estrattivi e salini. Tutto sommato non si può parlare di un grosso processo industrializzante. Si ebbe però un certo sviluppo della viabilità. A partire dal secondo e terzo decennio del secolo si ebbero dei risvolti positivi anche in ambito demografico, ed un'aumentata urbanizzazione. ${ }^{11}$

Le epidemie che all'epoca colpirono la penisola evidenziarono ancora una volta i limiti del sistema sanitario istriano e le debolezze della struttura sociale peninsulare. Le oscillazioni climatiche influirono gravemente sulla comparsa di una serie di malattie epidemiche. Le estati secche accompagnate da primavere ed autunni piovosi, grandinate ed inverni rigidi, oltre che carestie alimentari, favorirono la diffusione di malattie che colpirono in modo particolare gli strati sociali più deboli e malnutriti della popolazione. La malnutrizione li rendeva di conseguenza mal resistenti e li privava della necessaria immunità. Si generò una sorta di circolo chiuso. La carenza alimentare colpiva la popolazione e di riflesso la stessa agricoltura e gli altri settori economici, venendo a mancare la manodopera necessaria alla sua coltivazione. Ricomparvero le paure epidemiche, le carestie, le malattie agricole, si ebbe qualche risvolto demografico negativo, si accentuò la pressione fiscale, e si mostrarono delle tendenze migratorie. ${ }^{12}$

11 Apollonio, Libertà autonomia nazionalità; Marco Cuzzi - Guido Rumici - Roberto Spazzali, Istria Quarnero Dalmazia. Storia di una regione contesa dal 1796 alla fine del XX secolo, Trieste - Gorizia 2000, 41; Istria nel tempo, 484-497; Bernard Stulli, Istarsko okrǔ̌je 1825 - 1860, Pisino - Fiume 1984, 49-56 e 165; Prvi moderni popis stanovništva u Istri / Il primo censimento demografico moderno in Istria / Prvi moderni popis prebivalstva v Istri, a cura di Aleksej Kalc, Capodistria 2012.

12 Per affrontare la malattia furono esperimentati vari rimedi. Cfr. alcuni suggerimenti nell'ambito della rubrica "Cose del giorno", L'alchimista, a. VI, n. 33, Udine, 12 agosto 1855, 260-262. 
Tutto il quinto decennio del secolo e gli inizi di quello successivo erano contrassegnati da tutta una serie di scompensi climatici e naturali, terremoti e carestie, che generarono scardinamenti in primo luogo nel campo agricolo, sanitario e sociale. ${ }^{13}$ Il periodo coincide con la fine della cosiddetta Piccola età glaciale, che caratterizzò il continente europeo dalla metà del XVI secolo al 1850 circa, quando la temperatura terrestre iniziò ad elevarsi. ${ }^{14}$

Questa situazione mise a nudo i problemi strutturali dell'economia agricola, dell'evoluzione del sapere tecnico e scientifico, della mancanza d'investimenti e di capitale finanziario, del commercio, ecc.

$\mathrm{Ci}$ si rese conto della necessità di migliorare le condizioni dei terreni per aumentarne la fertilità, e delle aziende e degli impianti agricoli per renderli più concorrenziali. Occorreva investire nelle concimazioni, fin troppo legate all'insufficiente quantità di stallatico animale disponibile, modificare il sistema delle arature e delle rotazioni agrarie. Bisognava intervenire con altri fertilizzanti di sostegno e con nuovi tipi d'aratro e d'aratura. In Istria non si praticava ancora la cosiddetta aratura profonda. L'intervento aratorio era limitato ai pochi centimetri di superficie, mentre la scienza agronomica proponeva la diffusione di arature più profonde e degli aratri ad essa necessari. Un dissodamento autunnale più profondo del suolo avrebbe compensato la produzione. Ma gli aratri allora in uso nelle campagne erano inadatti a ciò. Per cui si doveva procedere con una seconda aratura sul solco ancora aperto, con la sua zappatura, o con la diffusione di aratri più pesanti, quali potevano essere quelli americani. ${ }^{15}$

Altre cause andavano ricercate nella mancanza di spirito capitalistico, industriale e commerciale, nel basso grado di alfabetizzazione di sviluppo civile nelle campagne.

Infatti, circolavano allora libri, giornali e periodici agricoli in cui si poteva attingere gli insegnamenti agricoli necessari, le informazioni relative a qualsiasi tipo di innovazione colturale. Però se da un lato non ci si preoccupava di diffondere questo sapere, dall'altro c'era un diffuso e preo-

13 Giacomo Braun, "Notizie meteorologiche e climatologiche della regione Giulia (Trieste, Istria e Friuli orientale)", Consiglio nazionale delle ricerche 2. Ricerche sulle variazioni storiche del clima italiano, Istituto di Geografia Fisica della R. Università di Padova, Roma 1934, 57-59.

14. Hrvoje Petrić, "Neke bilješke o »Malom ledenom dobu« (s malim osvrtom na Istru)" ("Alcuni appunti sulla «Piccola età glaciale» [con un piccolo riferimento all'Istria]”), Zbornik javnih predavanja, 2, Posebna izdanja 28 (Atti delle lezioni pubbliche 2, Edizioni particolari 28), Pisino 2013, 149-159.

15 "Sulle condizioni dell'agricoltura in provincia", La Provincia dell'Istria, a. XV, n. 24, Capodistria, 16 dicembre 1881, 185-186. 
ccupante analfabetismo. "Chi mai potrebbe credere che, a fronte di tanta luce di scienza agraria, e nei tempi delle universali ed agricole esposizioni, delle associazioni e dei congressi agrari, e poderi modelli, e cattedre ambulanti, e libri e giornali agrari circolanti in gran quantità, ecc... l'agricoltura nella gran maggioranza degli aratori sia tuttora il porto ingrato della miseria dell’ignoranza?! Eppure così è, lo si riconosca ad onore del vero.”16

Ciò cozzava col progresso oramai in atto nell'ambito della Monarchia asburgica. Nell'aprile 1864, nel suo discorso d'inaugurazione della prima seduta annuale della Dieta provinciale istriana, il commissario governativo Carlo conte Coronini di Cronberg, esaltò la politica agraria asburgica dei decenni precedenti, sottolineando l'aumento delle superfici agrarie, essendosi quadruplicata la coltura dei campi. ${ }^{17}$

L'alto esponente governativo esaltò le novità dei tempi moderni, indicando nella struttura fondiaria d'impronta medievale, "ibrida, incerta contrastata”, la causa dell'arretratezza economico-agricola istriana, alleviata dal recente "cangiarsi in proprietà civile, libera, piena, toglievansi le giurisdizioni baronali, divenute incompatibili." Il Coronini sapeva che senza un'adeguata istruzione nonché ammodernamento non c'era futuro per l'agricoltura provinciale. "Migliorate adunque la coltura dei campi, le gregge ed i boschi, perfezionate i vostri vini, completate il setificio”. Bisognava investire pure nella viabilità, per avvicinare i mercati e migliorare i rapporti tra l'entroterra e la costa, per cui egli invitava a moltiplicare "le comunicazioni avvicinando i villaggi e le terre alle città, e raccostandovi alle province interne dell'Impero”. Parole chiare, ma anche dure le sue, che illustrano chiaramente la problematica e l'evidente debolezza del sistema agrario provinciale, e le perduranti difficoltà d'ammodernamento.

Ma il vero problema stava in chi nella penisola deteneva sia il potere politico che economico, ovvero nella classe dei possidenti. Erano loro infatti che dovevano investire nel sapere agrario, da cui potevano ricavare i profili professionali ben preparati ed istruiti, capaci di dare nuovi impulsi alle loro proprietà. Erano loro dunque che dovevano accettare e promuovere il progresso e rispondere alle aspettative sociali.

In quanto ad innovazioni, si svilupparono la meccanizzazione agraria e la scienza agronomica, si diffusero le scuole agrarie, si organizzarono vari

\footnotetext{
16 "I Poderetti - scuola. Ordinati secondo il sistema Pestalozzi - Fellemberg", La Provincia dell'Istria, a. IV, n. 10, 16 maggio $1870,527$.

17 "Resoconto della prima seduta svoltasi il 6 aprile 1864", Atti della Dieta provinciale istriana, Parenzo 1865, 1.
} 
seminari d'aggiornamento e tavole rotonde, si stamparono e si diffusero libri e periodici d'agricoltura. Anche i parroci, dai loro pulpiti, contribuirono alla diffusione del sapere agrario. Le accademie continuarono pure con il loro apporto scientifico. Si diffusero con successo le varie associazioni di categoria, e comparve pure il capitalismo agrario. Si diffusero nuove colture, il girasole, con il suo derivato olio, e l'ailanto, che poteva dare nuovo impulso ai settori boschivo e serico. La bachicoltura ebbe un suo rinnovato impulso fino alla comparsa dell'atrofia. S'iniziò a prestare nuova attenzione anche al bosco. Ciò soprattutto a seguito delle devastazioni a cui fu soggetto negli anni di crisi 1852-63.

Si diffuse pure l'uso delle attrezzature metalliche, legato soprattutto alla diffusione delle strade ferrate. La costruzione delle ferrovie consentì infatti l'uso e la massiccia presenza del metallo fra la popolazione. In questo contesto, la diffusione dell'aratro metallico incarna uno dei miti maggiormente legati all'innovazione ed alla presenza asburgica nella penisola. La sua introduzione è stata accolta con sospetto. Esso ebbe una prima diffusione lungo la fascia costiera dell'Istria occidentale. Fu soprattutto l'Istituto agrario parentino ad impegnarsi in merito. ${ }^{18}$

\section{Sviluppo dell'istruzione agraria. La Scuola di viticoltura, enotecnia e pomologia di Parenzo}

Il processo di rinnovamento e di reimpostazione dell'economia agraria passava anche attraverso lo sviluppo dell'istruzione. Non si poteva infatti trasmettere il sapere agrario senza la diffusione dell'alfabetizzazione, delle scuole popolari e dei corsi d'agricoltura ad esse abbinate, della scuola agraria e di una società di categoria. Da qui l'avvio, fin dalla metà degli anni Cinquanta, di un dibattito le cui conseguenze furono a lungo andare l’inaugurazione della Società agraria, del Consiglio agrario provinciale, dell'Istituto agrario e della scuola agraria parentina, dei corsi d'agricoltura tenuti dagli insegnanti ambulanti, la diffusione delle banche agrarie. ${ }^{19}$ Le autorità regionali si accorsero che affinché il processo d'innovazione e di progresso abbia successo, era d'obbligo diffondere l'istruzione elementare, procedere allo sviluppo intellettuale ed alla formazione di maestri ed insegnanti.

18 Lidija Nikočević - Nevena Škrbić, "Način gledanja: Mit o Austriji u Istri” ("Angolatura: Il mito dell'Austria in Istria"), Istra: različiti pogledi. Etnografske zbirke Istre kroz austrijsko-hrvatski dijalog (Istria: angolature diverse. Le collezioni etnografiche istriane attraverso il dialogo austriaco-croato), Pisino 2001, 73.

19 Archivio di stato di Trieste (in seguito: AST), "I. R. Luogotenenza del Litorale. Atti generali (18501906)", b. 100 , fasc. $2 / 3-4$. 
Ciò soprattutto nelle campagne, dove mancavano scuole e maestri. Talvolta l'istruzione era portata avanti da sacerdoti inadatti o d'origine straniera e non conoscenti quindi la lingua d'uso nei villaggi. Da qui l'idea di avviare un corso d'istruzione pedagogica presso la scuola normale di Capodistria. ${ }^{20}$

Nel 1871, la Giunta provinciale approvò, dopo un periodo di stasi e di discussioni, una Risoluzione per l'istituzione della scuola agraria. In quello stesso anno, venne approvato un mutuo dal valore nominale di 100 fiorini a titolo di obbligazioni di Stato verso il Fondo confraternite ex venete, allo scopo d'istituire tale scuola. L'anno seguente, essa approvò la Delibera della Dieta concernete l'impiego del Fondo provinciale di cultura, e l'assunzione del sopracitato mutuo. ${ }^{21}$

Dopo aver ottenuto l'assicurazione che alla sua creazione avrebbero partecipato anche i sussidi di Stato, si procedette alla ricerca di un maestro - direttore qualificato e capace a guidare l'istituto, e si offrì in vendita una tenuta campestre ritenuta adatta alle esigenze scolastiche. Gli utensili necessari all'attività, andavano invece richiesti alle varie Società agrarie. Nel 1874, venne finalmente fondata la Stazione provinciale modello di viticoltura e di governo delle cantine, con annessa sezione pomologica, con a capo, dal mese di febbraio, il cav. Emilio von Mayersbach. ${ }^{22}$ Detta istituzione, con sede a Parenzo, disponeva di un campo, e di un laboratorio fornito dell'indispensabile, con 18 tipi di vino, di cui 10 originati da uve nostrane. Finalmente, l'8 febbraio 1883 aprì battenti la Scuola di viticoltura, enotecnia e pomologia di Parenzo. ${ }^{23}$

Ma aprire la scuola non bastava. Le speranze che i suoi sostenitori e gli intellettuali ponevano in essa erano molte. Occorreva però prevenire un altro grosso problema: la possibilità o meno, per i giovani diplomati, di trovare un adeguato impiego professionale presso le aziende agricole in mano alla proprietà terriera. E l'atteggiamento degli agiati, favorevole agli studi, dava ben sperare per il progresso agrario. ${ }^{24}$

\footnotetext{
20 Idem, b. 124, fasc. 2/4-5.

21 "Relazione generale alla Dieta provinciale del Margraviato d'Istria sulla gestione della Giunta provinciale dalla sessione dietale dall'anno 1871 in poi" (in seguito: "Relazione generale"), Atti della Dieta provinciale istriana, Parenzo 1871, 17.

22 Idem, 14.

23 Denis Visintin, "Istria, gli inizi della viticoltura moderna”, La Voce del popolo, http://www.editfiume.com/ lavoce/cultura/3481-istria-gli-inizi-della-viticoltura-moderna; Elena Poropat Pustijanac, Polje znanja. Povijest porečke poljoprivredne znanosti i obrazovanja / Il campo del sapere. Storia della scienza e dell'istruzione agraria a Parenzo, catalogo della mostra, Parenzo 2013, 14.

24 La Provincia dell Istria, a. VII, n. 22, 16 novembre 1873, 1357-1358.
} 
Fu grazie all'attività dell'Istituto parentino, che il vino istriano ebbe una sua ulteriore diffusione all'estero. Grazie ad esso, i viticoltori istriani iniziarono ad acquisire sapere ed esperienze internazionali, con la presenza alle esposizioni internazionali di categoria. I primi risultati non erano certo soddisfacenti, ma col tempo questo tipo di confronto e di esperienze provocherà degli effetti futuri decisamente positivi.

Contemporaneamente, in quegli stessi anni, la Giunta approvò tutta una serie di decreti tesi ad alleviare le condizioni agricole. Primi fra tutti, la Legge sulla sicurezza delle possessioni campestri, il progetto di Legge di bonifica delle aree paludose, il "Progetto di legge sulla fabbricazione e la commercializzazione dei vini artificiali” (all'epoca ancora non diffusi nella penisola), e si stanziarono pure degli stipendi per gli interessati ai corsi di studio sulla lavorazione del vino e l'arte del cantinaggio, l'enologia e l'oleificazione. S'introdussero anche i libri delle ipoteche. Venne prestata particolare attenzione alla regolazione dei corsi d'acqua. ${ }^{25}$

Si dette inoltre spazio alle iniziative promozionali tese alla diffusione della coltura olearia, ed all'incremento della produzione dell'olio da tavola.

Grande fu anche in questo contesto il ruolo svolto dall'Istituto agrario parentino, che sotto la guida dei direttori Hugues e Cucovich diedero vita ad una grande scuola sperimentale presso la tenuta dei Filippini di Cittanova. ${ }^{26}$ L'intensa attività di studio che si generò in quegli anni attorno alla piante permise di scoprirne nel triennio 1873-75 le tante varietà, a loro volta soggette ai diversi mutamenti da località a località e da comune a comune. ${ }^{27}$

Nonostante tutti questi sforzi, iniziò una fase discendente per tale coltura, al punto che agli inizi del secolo successivo l'olio d'oliva istriano veniva usato come puro e semplice olio da macchina. Diminuirono anche i terreni riservati a tale coltura, a favore di quelle granarie.

Ancora, come nei secoli precedenti, la produzione subiva terribili ed altalenanti alti e bassi, alternando ai momenti di prosperità altrettanti momenti di crisi dovuti alle gelate che per quattro volte colpirono le colture istriane nel corso del secolo. ${ }^{28}$

25 "Relazione generale", 18.

26 Mario Zanini, "Una storia istriana di mille e mille anni. Olivo, ulivo, ulì, uleio. Torchi, frantoi, olio”, Ricordando Cittanova, Trieste, aprile 2006, 11.

27 Ibidem.

28 Ibidem. 
Per affrontare queste sfide dovute alla liberalizzazione del mercato interno, si rese necessaria l'azione comune e l'unione dei piccoli e medi possessi, con cui si favorì la comparsa delle cooperative agrarie e delle cantine vinicole sociali, che ben presto iniziarono a promuovere i prodotti vinicoli, e ad organizzare le prime mostre enologiche e degustative. Tale azione si rese necessaria pure per affrontare la concorrenza esterna ed i prodotti artificiali combattuti dalla Monarchia mediante apposite leggi. ${ }^{29}$

\section{La lotta contro le malattie agricole}

Ma a scuotere la padronanza e le autorità è stata la lotta contro i parassiti e le malattie, indicando il reale stato di sviluppo del settore. Se da un lato molte epidemie e parassiti non ebbero conseguenze disastrose (tignola, cicalina, marciume delle viti, ecc.), dall'altro alcuni mali rischiarono di annientarla del tutto, e di trascinare nella rovina molte famiglie, con ricadute disastrose per tutta l'economia istriana, per cui le autorità provinciali affrontarono le problematiche con decisione ed energia.

Il primo pericoloso male che fece la sua comparsa fu l'oidio o crittogama della vite, di origine nordamericana, scoperto nel 1852 sia in Europa che in Istria, che per un decennio intaccò numerosissimi vigneti. Nella penisola, l'attacco maggiore lo si ebbe nel $1859 .^{30}$ Questo attacco generò una prima sorta di rivoluzione tecnico scientifica nell'agro istriano. La viticoltura europea, e di conseguenza quella istriana, fu salvata dall'introduzione di nuove viti americane immuni, e dall'intervento chimico-scientifico. L'avvento della solforazione, e la conseguente diffusione delle macchine solforatrici, rappresentarono l'inizio di una vera e propria rivoluzione. ${ }^{31}$

Decisivo fu l'intervento della Dieta provinciale che finanziò il suo acquisto e la distribuzione nei comuni infetti. Iniziò allora un periodo di ripresa e di diffusione della viticoltura istriana, che durò per circa un ventennio, favorito dall'impianto di nuove viti a sostituzione di quelle danneggiate, ma anche per estendere i vigneti, si ebbe la ripresa. ${ }^{32}$

29 “Legge contro i vini artificiali”, L'Istria agricola, n. 1, Parenzo, novembre 1907, 2-6.

30 Denis Visintin, "Kratki osvrt na povijesni razvoj vinarskih podruma početkom XX. st." ("Breve contributo allo sviluppo storico delle cantine vinicole in Istria all'inizio del XX secolo”), Stoljeće vina 1901. - 2001. Doprinos kulturi vina u Istri (Il secolo del vino 1901-2001. Contributo alla cultura del vino in Istria), Pisino 2001, 63; idem, "Način gledanja: austrijska poljoprivredna politika” ("Angolatura: la politica agraria austriaca”), Istra: različiti pogledi, 85; Carlo De Franceschi, Descrizione del Margraviato d'Istria, Parenzo 1879, $224-225$.

31 "Relazione della Commissione provinciale sulla provvista e sulla distribuzione dello zolfo per le viti" (in seguito: "Relazione della Commissione provinciale"), Atti della Dieta provinciale istriana, Parenzo 1863 , 581-587; De Franceschi, Descrizione del Margraviato d'Istria, 116, 124 e 138.

32 "Relazione della Commissione provinciale", 581-587; De Franceschi, Descrizione del Margraviato d'Istria, $95,116,124$ e 138. 
Particolarmente critico fu il 1853. Alle devastazioni crittogamiche, si unirono i magri raccolti cerealicoli e particolarmente del granoturco. Scarsi pure i legumi, i foraggi e le olive. In pratica, furono colpiti tutti i settori primari a cui la popolazione, in primo luogo quella agricola, poteva fare affidamento. Ciò in conseguenza di una serie di furiose grandinate e della siccità. I riflessi sui settori commerciali delle città - piazze di mercato, Trieste compresa, ebbero pure delle ripercussioni paurose. Le autorità delle principali città istriane richiesero un intervento straordinario dai fondi della Tesoreria di Stato per venire incontro ai bisogni della popolazione, ed evitare le possibili peggiori conseguenze d'approvvigionamento del futuro inverno. ${ }^{33}$ Di conseguenza, per sopravvivere ci si rivolse a quanto altro Madre natura poteva offrire. Aumentò allora il taglio del legname nei boschi e la vendita degli animali grossi utili al lavoro nei campi. E si ebbe pure una vendita stracciata degli immobili. ${ }^{34}$ La posizione dei contadini fu resa ancor più difficile dai gravami dovuti al processo di esonero del suolo, conseguente all'abolizione degli oneri feudali, che si aggiunsero alla serie di gravami, molti dei quali inevasi e con forti arretrati a carico dei contribuenti. Le conseguenze più gravi furono sentite nell'agro di Canfanaro, in quello di Isola e nel Distretto di Parenzo, colpiti da un paio d'anni dalle calamità naturali. ${ }^{35}$ Le autorità reagirono con decisione, soprattutto laddove la vita della popolazione dipendeva esclusivamente dai prodotti della terra. Tale era il caso di Canfanaro, di Racizze e di Grimalda per esempio, ma anche dell'entroterra parentino, dove molti furono ridotti alla fame, giungendo a nutrirsi di bacche e di radici per sopravvivere. Seguì l'invio degli aiuti che interessò anche il Lombardo - Veneto. Le precarie condizioni provinciali, e le scarne entrate erariali, avevano spinto la Dieta a rivolgersi all'Imperatore. I lunghi periodi di siccità, le piogge, le grandinate, le gelate, la presenza dell'oidio, l'atrofia dei bachi da seta, le malattie della patata e degli ulivi decimarono la produzione. Le famiglie contadine furono colpite dalla crisi e dalla miseria. Il mercato s'impoverì e vennero meno i mezzi necessari a far fronte alle spese correnti. Perciò

33 AST, "I. R. Luogotenenza del Litorale (1850-1918). Atti presidiali”, b. 13, fasc. 1/4-3.

34 Rino Cigui, "La crisi agricola degli anni 1860-62 nel distretto di Parenzo" (in seguito: "La crisi agricola”), La Ricerca, 55, Trieste - Rovigno, giugno 2009, 3.

35 AST, "I. R. Luogotenenza del Litorale (1850-1918). Atti presidiali“, b. 56, fasc. 1/4-3; Biskup Juraj Dobrila zastupnik naroda Istre u Istarskom saboru (1861.-1869.) i bečkom Parlamentu (1861.-1865.) (Il vescovo Giorgio Dobrila rappresentante del popolo dell'Istria, alla Dieta istriana [1861-1869] e al Parlamento di Vienna [18611865]), a cura di Ivan Jelovac e Mladen Juvenal Milohanić, Pisino 2008, 200. 
vennero meno le entrate erariali, ed il denaro necessario alla gestione delle autonomie comunali. ${ }^{36}$

Il male provocò preoccupazione e sconcerto tra la popolazione, visto che si colpiva la sua industria di maggior profitto. In più d’un decennio, trascorso in gran parte senza sperimentare alcuna soluzione, furono devastati numerosi vigneti. Le conseguenze furono disastrose anche in campo commerciale, visto che il mercato regionale si rivolse ai vini carniolici ed ungheresi. Di conseguenza, la Giunta provinciale provvide all'applicazione dello zolfo, in precedenza sperimentato con successo nel levante italiano e nel goriziano. Esso rappresentava una costante ed una minaccia continua, soprattutto per le famiglie di minore forza economica. Per cui fin dalla fine del 1861, essa deliberò l'acquisto di tale rimedio e degli istrumenti adatti all'uso, sovvenzionando i possessori. A tale caso si anticiparono i fondi dal Sovrano Erario. Ovviamente, visti i pregiudizi della contadinanza, non tutto filò liscio. All’iniziativa non aderirono molti privati e diverse autorità locali. Si ebbero invece adesioni ed esiti eccellenti nei distretti di Capodistria, Pirano, Buie, Parenzo, Pola, Pisino, Albona. All'azione aderirono pure il vescovo di Parenzo e Pola mons. Dobrila e numerosi sacerdoti. Soltanto con gli interventi solforativi, e con l'impianto di nuove viti a sostituzione di quelle danneggiate, ma anche per estendere i vigneti, si ebbe la ripresa. ${ }^{37}$

Si trattò però anche qui di una lotta lunga contro i pregiudizi e le superstizioni. Infatti, ancora nel 1890, quindi a quasi tre decenni di distanza dalla diffusione dello zolfo, s'invitava i Consorzi agrari a non insistere a proporre rimedi inutili, e ad attendersi "ai lumi offerti dalla pratica e sistematica sperimentazione in Provincia, ben accertati dal Consiglio (agrario provinciale, n.d.a.), riponendo fiducia nei metodi da questo raccomandati." Tali difficoltà d'affermazione del nuovo che avanzava erano spesso denunciate dalle autorità.

Le autorità intervennero stanziando denaro per l'acquisto, organizzando la distribuzione organizzata delle granaglie in vari punti della penisola, ed approvando misure di sollievo varie, quale ad esempio l'inesigibilità di tutte le tasse non riscosse. Numerosi furono gli appelli alla generosità

\footnotetext{
36 "Resoconto della IX seduta svoltasi il 29 gennaio 1863", Atti della Dieta provinciale istriana, Parenzo 1864, 54 .

37 "Relazione della Commissione provinciale", 581-587; De Franceschi, Descrizione del Margraviato d'Istria, $95,116,124$ e 138.

38 "Misure contro la peronospora viticola", La Provincia dell Istria, a. XXIV, n. 1, 1 gennaio $1890,4$.
} 
degli abitanti dei territori vicini, Trieste ed il Quarnero in primo luogo, che risposero con l'invio di aiuti e l'organizzazione di serate di beneficenza. Alle esigenze istriane risposero pure alcuni comuni ed uffici parrocchiali del vicino Friuli. ${ }^{39}$ Le autorità locali allora procedettero con l'acquisto di frumento e granoturco, distribuito in vari punti della penisola. ${ }^{40}$

Agli inizi degli anni Settanta i prodromi della crisi erano ancora evidenti, seppur si notava una certa ripresa. Gli affanni atmosferici avevano causato una riduzione della fioritura viticola, ma la crittogama della vite era stata posta sotto controllo, almeno nelle aree più progredite. Parte della produzione, colpita dalla tempesta, era stata salvata con la vendemmia prematura, e molti erano stati gli impianti nuovi introdotti per sopperire ai vitigni colpiti dalla malattia. "Quantunque scarso il prodotto, in confronto di questi ultimi anni si fa molto vino e se ne farà molto di più appena vengano su tutti i nuovi impianti."

In effetti, se un certo progresso lo si poteva notare nei distretti più avanzati, quali quelli di Capodistria, Buie o Parenzo, non dappertutto era così. L'anonimo corrispondente della Provincia dell'Istria di Pisino, constatava nel 1877 che si era ancora molto lontani dal progresso agrario promosso dall'opinione pubblica. Uno degli ostacoli andava ipotizzato nell'ancora presente e diffusissima coltura mista: segno evidente questo dell'ancora generale assenza di impianti specializzati e di un'agricoltura di tipo industriale. Mancava ancora un'adeguata cultura istruttiva di cantinaggio e di lavorazione dell'uva. Se a Pirano, a Buie, a Montona, a Capodistria, ad Albona l'uva portata a maturazione era matura, in buona parte dell'Istria l'uva la si lavorava ancora acerba. Molto spesso questa prematura ed inopportuna vendemmia era la diretta conseguenza dei furti campestri che ancora si praticavano nelle campagne capodistriane ed in buona parte degli altri distretti peninsulari. L'istituzione di impianti specializzati, facilmente sorvegliabili, e di cantine vicine ai vigneti forse avrebbe ovviato almeno parzialmente questo problema. La vicinanza degli impianti di lavorazione avrebbe favorito una minore schiacciatura dell'uva durante il trasporto. Insomma, qualsiasi piccolo accorgimento lavorativo sarebbe risultato salutare. ${ }^{42}$

39 AST, "I. R. Luogotenenza del Litorale (1850-1918)", b. 13, fasc. 1/4-3; Cigui, "La crisi agricola”, 3.

40 AST, “I. R. Luogotenenza del Litorale (1850-1918)", b. 13, fasc. 1/4-3.

41 La Provincia dell Istria, a. IV, n. 20, 16 ottobre 1870, 605.

42 “Corrispondenze”, La Provincia dell Istria, a. XI, n. 11, 1 giugno 1877, 83. 
Nel 1878, le autorità provinciali sottolinearono amaramente come le continue piogge ed i trascorsi inverni rigidi avevano rovinato un po' tutte le produzioni, portando molte famiglie contadine sull'orlo della miseria. Di conseguenza, la Giunta provinciale richiese alle autorità centrali ulteriori interventi d'approvvigionamento per quei piccoli produttori che, imbavagliati dai costi di produzione, dalle spese e dai crediti, si trovavano con l'acqua alla gola. Il biennio 1879-80 fu uno dei peggiori nella storia agraria del periodo. Gli eventi meteorologici portarono desolazione, miseria e carestie in tutta la penisola.

Nella seconda metà degli anni Settanta comparvero il vajuolo della vite, la filossera (scoperta però soltanto nel 1880), le cavallette, la clorosi. ${ }^{43}$ Si diffuse pure il tarlo dell'uva, il Rinchite e Tortiglione, e l'othyorhynchus o ladrone, e la clorosi, che ostacolava l'innesto delle viti americane. Intorno agli anni Ottanta si diffuse anche la peronospora. ${ }^{44}$

Inizialmente, quest'ultima non preoccupò molto i vitivinicoltori istriani, in quanto le condizioni climatiche non erano eccessivamente favorevoli al suo sviluppo. Quasi contemporaneamente, essa si diffuse nel vicino Friuli. ${ }^{45}$ La situazione peggiorò negli anni 1884-85, complice l'ignoranza della contadinanza in materia e l'inefficienza dei preparati chimici a disposizione. In conseguenza di ciò, e tenendo presente pure l'attacco fillosserico oramai in atto, si ebbe una paurosa flessione produttiva. Gli agricoltori intervennero con delle aspersioni di latte di calcio, che generarono ulteriori danni al fogliame a causa del brusone e delle scottature a cui furono soggette. Laddove s'intervenne con la miscela di solfato di rame e calce, i risultati furono decisamente soddisfacenti. ${ }^{46}$ La problematica interessò anche il Consiglio agrario provinciale, il quale mise a disposizione dei Consorzi agrari e dei possidenti rilevanti quantità di poltiglia bordolese, ammoniuro di rame e miscele cupro-calciche, distribuite particolarmente nei territori

$43 \mathrm{Si}$ vedono a questo proposito le varie relazioni della Giunta regionale, e gli interventi dei membri della Dieta negli anni 1878, 1880, e 1881. Cfr. inoltre Giovanni Bolle, "L’infezione fillosserica in Istria nel 1886", Atti della Dieta provinciale istriana, Parenzo 1887, LVI.

44. Idem, LVII.

45 Paola Ferraretto, Caso studio per la valorizzazione e preservazione di vecchie varietà di uva a bacca rossa del Friuli-Venezia Giulia: il vin di Uchi (in seguito: Caso studio), tesi di laurea, Università degli studi di Udine, a. a. 2007-2008, 6 .

46 Vido Vivoda, "Povijest vinogradarstva Istre od 1841. do 1941. god." ("Storia della vitivinicoltura in Istria dal 1841 al 1941"), Gospodarstvo Istre. Časopis za gospodarska pitanja (L'economia dell'Istria. Rivista per le questioni economiche), a. 1, n. 2, Pola 1988, 13; "Sulle condizioni agricole del territorio di Pirano e il compito dei piranesi di fronte all'infezione fillosserica", La Provincia dell Istria, a. XX, n. 22, 10 novembre 1886, 169-170; idem, a. XX, n. 23, 1 dicembre 1886, 177-178; Bolle, "L'infezione fillosserica in Istria nel 1886", LVIII. 
di Albona, Buie, Capodistria, Dignano, Montona, Pirano, Pisino e Pola, nonché macchine ed apparecchi vari. ${ }^{47}$

Al contrario della peronospora, la fillossera produsse effetti e paure ben più devastanti per tutta la vitivinicoltura europea, nonché innovativi scardinamenti e trasformazioni, scomparendo per sempre molte qualità di viti che si rifacevano all'antica vitis vinifera europea. Tale distruzione su vasta scala fu dovuta ad un insetto di cui all'inizio non si conoscevano ne le forme di sviluppo, ne la biologia, ne le modalità di diffusione, il che ne favorì lo sviluppo in tutto il continente europeo.

Il parassita si nutriva sia delle radici che delle viti, distruggendo completamente i vigneti intaccati. Gli esperti notarono che sulle viti americane esso intaccava le foglie formando delle galle, entro la quale si sviluppava la prole, parte della quale colpiva le radici. Sulle viti europee le femmine non riuscirono a formare le galle sulle foglie, per cui il ciclo di vita dei parassiti si ridusse ad una serie di generazioni viventi sulle radici, che marcivano e portavano a morte la pianta.

In quanto all'origine, esso è stato introdotto in Europa con delle barbatelle di viti americane, dato che alcune varietà erano presenti in Europa già intorno alla metà del terzo decennio del XIX secolo. Esse ebbero una particolare e fortunata diffusione in Francia negli anni 1852-54, nelle zone colpite dall'oidio. Infatti, le barbatelle americane si presentavano assai ben resistenti a tale malattia, per cui si pensò d'importarne altre per contrastare l'emergenza. Tale operazione avvenne in diversi stati europei. Tali introduzioni interessarono diversi stati europei: Francia, Regno Unito, Irlanda, Germania. L'infezione si manifestò innanzitutto in Francia - dove fu scoperta nel 1865 ed individuata tre anni dopo - la maggiore usufruttuaria delle barbatelle americane infette. Dopo vari tentativi inutili, si giunse alla soluzione del problema, decidendo di contrastarlo con la stessa arma che l'aveva provocata: l'introduzione, ancora una volta, di viti americane. L'intuizione la si deve al prof. Jules Émile Planchon di Montpellier (18231888) che dopo aver individuato l'origine americana della fillossera, capì che la lunga convivenza con il parassita aveva permesso alle viti americane di diventarne immuni. L'immunità infatti permetteva di dare vita ad una nuova pianta bimembra. Tale scoperta rivoluzionò l'agricoltura europea, facendo entrare in scena la scienza ed aprendo le porte al connubio tra essa

$47 \mathrm{Si}$ vedano a questo proposito le varie Relazioni sull'attività svolta presentate annualmente dal Consiglio agrario provinciale. 
e l'economia agricola. La nascente pianta presentava un portainnesto con radici di vite americane su cui s'impiantavano i vitigni europei. Si manifestò allora una fulminea corsa alla ricerca delle varietà americane affini sia all'innesto che ai suoli europei. Si era pure di fronte ad una nuova tecnica di coltura della vite: l'innesto. Iniziò allora un immenso lavoro portato avanti con difficoltà causa l'ignoranza, le superstizioni e le scarse cognizioni agrarie dei vitivinicoltori. Da questa lotta la viticoltura uscì completamente trasformata. La comparsa delle sopra menzionate avversità ha causato non tanto un'evoluzione, quanto una vera e propria rivoluzione nella tecnica stessa della lavorazione viticola: non bastava più applicare le tradizionali norme demandate da padre in figlio, ci si doveva appoggiare oramai sulle scienze biologiche e fisico-chimiche, che stavano diventando indispensabili per lo sviluppo viticolturale. Quasi tutta la viticoltura europea fu ricostruita con le nuove barbatelle bimembre, e la viticoltura antica tradizionale lasciò il passo a quella nuova, di marca scientifica.

Prima di ciò, per contrastarne la diffusione, si intrapresero molte strade: l'uso del solfuro di carbonio iniettato nel terreno per uccidere le gallicole infette sulle radici, la sommersione dei vigneti per distruggere per asfissia quelle ibernanti (praticabile solo in zone pianeggianti e irrigabili), l'insabbiamento delle vigne, possibile soltanto nei terreni sabbiosi d'origine marina.

Nei territori asburgici, essa venne scoperta nel 1874 nei fondi della Scuola di Viticoltura di Klosterneuburg, in Austria. Nel 1888 essa comparve nel Goriziano, ${ }^{48}$ e gradualmente si diffuse nei territori del Collio.

Una prima decisa reazione delle autorità centrali la si ebbe con l'emanazione della Legge dell'Impero 3 aprile 1875. Essa definiva che l'indennizzo complessivo con cui venivano remunerati i possidenti danneggiati doveva venir ripartito fra di essi in proporzione all'imposta fondiaria erariale prescritta l'anno precedente. Gli indennizzi potevano essere assunti a carico del Fondo provinciale, a patto che le Diete regionali s'esprimano positivamente. Detta legge obbligava inoltre i proprietari dei vigneti a denunciare alle autorità eventuali sospetti concernenti la comparsa dell'infezione fillosserica. E fu proprio grazie a ciò che vennero scoperti molti focolai infetti. Ma allora, per la Dieta istriana, il pericolo era ancora lontano, visto che la fillossera - diffusa dal 1872 ma identificata soltanto nel 
1880 nella penisola - era circoscritta ai vigneti di Klosterneuburg, Alsegg e Nussdorf, nell'Austria Inferiore, ed ai dintorni di Pančevo (ung. Pancsova, ted. Pantschowa), nel Banato ungherese.

Stando all'inchiesta avviata per scoprire le origini dell'epidemia nella penisola, pare che ad Isola essa si fosse manifestata nel 1872, ed a Cortina e nella valle di Sicciole (Pirano), due anni dopo. ${ }^{49}$

Grande fu lo sgomento e lo stupore dei proprietari, che inizialmente affrontarono con molta leggerezza l'epidemia, convinti che soltanto con assidue e costanti cure, si potesse combattere il parassita. Purtroppo le loro previsioni furono sbagliate, e le conseguenze disastrose, provocando un pauroso arresto in questo settore, che vedeva l'area contaminata primeggiare in quanto a progresso, nella prima parte della seconda metà del secolo. I proprietari infatti si diedero alla specializzazione esclusiva dei terreni in vigneti, con degli impianti in linea, coltivazioni a palo secco ed a ceppo unico, concimati, potati scacchiati, cimati, diligentemente curati e provvisti di canali di scolo, con largo uso di zolfo per combattere l'oidio. La produzione vitivinicola era in aumento, ed aumentava il benessere economico. I danni arrecati si fecero sentire anche a livello economico: diminuivano la produzione sia dell'uva che del vino, nonché la qualità dei prodotti, si abbassava il prezzo enologico di mercato, aumentava la concorrenza mercantile dei vini prodotti nella bassa Istria - immune dall'infezione fillosserica - e di quelli carniolici ed ungheresi.

Quando venne identificata, la fillossera aveva già allora una notevole e preoccupante diffusione. Infatti, subito in apertura della III seduta della Dieta provinciale istriana, fu annunciata l'apparizione della phyllossera vastatrix nel piranese, e si era manifestato il timore, purtroppo dimostratosi veritiero poco dopo, di una sua presenza pure nel territorio isolano. La Giunta provinciale prese allora degli immediati provvedimenti, ed incaricò il prof. Leonhard Roesler (1839-1910), della Stazione enologica di Klosterneuburg, ad ispezionare i territori e relazionare in merito. Con riferimento alla Legge dell'Impero del 3 aprile 1875, si propose un'anticipazione dal Fondo provinciale d'agricoltura degli importi necessari alle misure in essa previste contro la diffusione del "pidocchio della vite", fermo restando l'obbligo del risarcimento di tutti i possidenti di piantagioni viticole della Provincia. A

49 Sull'origine e la diffusione della fillossera nella penisola, cfr. "La Phylloxera vastatrix negli anni 1880 e 1881. Relazione di Giovanni Bolle Direttore dell'i. r. Istituto sperimentale di bachicoltura ed enologia in Gorizia", Atti della Dieta provinciale istriana, Parenzo 1881. 
loro volta, tali mezzi vennero anticipati dal Sovrano erario, con l'obbligo di restituzione dal Fondo provinciale. ${ }^{50}$

Si istituì pure un'apposita commissione provinciale che propose tutta una serie di iniziative per contrastarne la diffusione: il divieto d'importazione di nuovi vitigni, la diffusione delle nozioni circa la diffusione dell'epidemia e dei metodi con cui combatterla, l'organizzazione d'impianti sperimentali e di semenzai, l'uso sperimentale del solfato di rame - metodo quest'ultimo costoso ma efficace, per cui risultò molto più popolare l'impianto di viti americane, per diffondersi quindi nelle altre aree colpite, tra cui il buiese l'introduzione di seminari d'aggiornamento in materia di potatura delle viti. ${ }^{51}$

Al fine di limitare ed ostacolare l'infezione, le autorità provinciali invitarono le podesterie e la possidenza a non lasciarsi cogliere dal panico e dalla disperazione, né a sperare in un’immediata scomparsa della malattia, né ad abbandonare i vigneti al caso, né a trascurare quelle cognizioni che potessero essere importanti a scoprirla ed a debellarla. Si deliberò l'introduzione di viti americane nei territori infetti, da attuare di comune accordo tra il Governo centrale e le autorità provinciali e l'introduzione di appositi vivai in cui sperimentare la sostituzione delle viti indigene. La propagazione di vitigni resistenti alla filossera, quali quelli americani, riguardava per il momento soltanto i territori infetti, e la questione era di primaria importanza e rispondeva agli interessi economici provinciali. Prima di procedere però in merito, la Giunta volle tastare il polso dell'opinione pubblica, che peraltro non conosceva né la vera natura né i reali pericoli dell'epidemia, né la conseguente impotenza a contrastarlo. Sorsero eccezioni, opposizioni e diffidenze contro una introduzione di viti americane nelle località al momento immuni. Il Capitano provinciale dott. Francesco Vidulich si rivolse agli esperti in materia, il Barone de Babo, ed al dott. Roesler, direttori rispettivamente dell'i.r. Istituto eno-pomologico e della Stazione chimico-fisiologica di Klosterneuburg, ed al sig. Göthe, direttore della Scuola provinciale di frutticoltura ed enologia di Marburg, per avere un loro parere in merito. Essi si schierarono a favore dell'introduzione delle viti americane, la cui immediata applicazione permetteva un risparmio di fatica,

50 "Resoconto stenografico della terza seduta della Dieta provinciale istriana", Relazione generale alla Dieta provinciale del margraviato d'Istria sulla gestione della Giunta provinciale dalla sessione dell'anno 1880 in poi, 10.

51 Viktor Vitolović, "Razvoj vinogradarstva u Istri od 1860. do 1914. s posebnim obzirom na ekonomsko jačanje istarskih (hrvatskih) seljaka" ("Lo sviluppo della viticoltura in Istria dal 1860 al 1814 con particolare riferimento al rafforzamento dei contadini istriani [croati]”), Hrvatski narodni preporod u Dalmaciji i Istri, 483. 
tempo e denaro. Dal canto suo, il Ministero manifestò la sua contrarietà ad una loro introduzione, in attesa dei risultati degli esperimenti avviati a tale proposito in Austria. ${ }^{52}$

La Giunta provinciale, che esprimendo il suo disaccordo con la missiva ministeriale, ricordò gli esperimenti francesi dimostranti la loro longevità. La filossera aveva distrutto nel giro di un quinquennio al massimo tutte le viti europee nelle zone in cui si era diffusa e l'unica via di salvezza per le varie viticolture regionali minacciate stava nell'introduzione delle viti americane. Perciò essa richiese con fermezza l'abolizione, per un anno, del divieto d'introduzione delle viti americane in atto.

Le autorità provinciali vollero conoscere lo stato territoriale delle cose e verificare la reale diffusione del male. Si visitarono allora i territori comunali al momento ritenuti immuni: Villanova, Visignano, Caroiba, Montona, Grisignana, Momiano, Merischie, Buie, Castelvenere, Capodistria, S. Canziano, S. Bastiano, Sermino, S. Nicolò, S. Marco, S. Rocco, Muggia. Tali esplorazioni, se scagionarono la fillossera per lo stato di deperimento in cui versavano molti vigneti, permisero di scoprire la presenza di parassiti vegetali quali l'antracnosi o il vajuolo, i ladroni neri ed i tortiglioni (rhynchites betuleti). L'immagine che si presentava di fronte agli occhi degli ispettori molto spesso era quella di un paesaggio agrario desolatamente abbandonato a se stesso, in cui le condizioni del suolo, la trascuratezza e l'umidità erano le cause principali dei deperimenti.

Le esplorazioni degli esperti, effettuate nei periodi estivo ed autunnale degli anni 1881-82 nei territori fillosserati di Pirano ed Isola confermarono la preoccupante diffusione del male. La Giunta provinciale ritenne giusto l'abbandono della diretta ed immediata ingerenza delle autorità nella questione, sia per la vastità delle superfici infette, sia per le spese ingenti che si dovevano sopportare. Il parere degli esperti tendeva sempre più all'abbandono del metodo cosiddetto estintivo (estinzione delle viti infette), a favore di quello colturale (sostituzione delle viti). Tali interventi interessavano e colpivano direttamente i proprietari e la totale distruzione dei vigneti ad opera della malattia apportava danni incalcolabili. Motivo per cui anche gli interessi dei singoli e gli aiuti collettivi dovevano convergere

52 "Relazione della Giunta alla Dieta provinciale sulla avuta attività di fronte all'invasione filosserica in Provincia, e domanda d'istruzione sulla propria azione futura", Relazione generale alla Dieta provinciale del margraviato d'Istria sulla gestione della Giunta provinciale dalla sessione dell'anno 1881 in poi, Parenzo, 23 agosto 1881 . Si vedano anche gli allegati n. 1-8. 
verso la soluzione del problema. Per cui la Commissione provinciale antifilosserica invitò l'autorità politica distrettuale ad ordinare l'applicazione del sistema colturale ai singoli proprietari dei terreni fillosserati. Mancando l'approvazione ministeriale, si propose di anticipare i mezzi necessari dal Fondo provinciale, e di riservare quanto ancora rimaneva degli assegni statali destinati al procedimento estintivo agli interventi d'emergenza nel caso si manifestassero nuovi centri d'infezione. ${ }^{53}$

Tuttavia, l'uso dei trattamenti colturali andava incontro a determinate difficoltà. Alcuni dei materiali necessari dovevano essere importati dall'estero a gravami daziari ed a spese di trasporto notevoli. Le tardive consegne comportavano conseguenti ritardi nell'applicazione. Molti proprietari osteggiavano la cooperazione, dovendo impegnare la manodopera necessaria ai trattamenti antifillosserici nei lavori primaverili. Il maltempo, l'inesperienza, la mancata sperimentazione a livello locale, la condiscendenza verso i proprietari delle vigne infette, le superstizioni e le perplessità ci misero pure lo zampino. Perciò si decise di sperimentarli all’inizio del ciclo vegetativo, quando se ne potevano constatare gli effetti ed intervenire a proposito.

La preoccupante diffusione del male convinse la Commissione ad avviare anche successivamente, in via del tutto preventiva, delle esplorazioni anche nei comuni limitrofi alle aree infette. Nel 1882 esse diedero un esito negativo nei comuni di Corte d'Isola, Castelvenere, S. Pietro dell'Amata, Padena, Villanova e Buie, mentre diede esito positivo a Salvore. ${ }^{54}$

Nel giugno del 1883 si propose di estendere le esplorazioni al distretto politico di Parenzo, e d'abolire il divieto di reimpianto di viti site nei comuni censuari di Pirano ed Isola, già assoggettate al trattamento estintivo, e di mantenere in vigore tale proibizione per i vigneti posti in altri comuni, non ancora sottoposti a tale trattamento. ${ }^{55}$

Lanno dopo esse interessarono Pirano, Isola, Salvore, Corte d'Isola, Padena, S. Pietro dell'Amata, Villanova, Castelvenere, Buie, Matterada, Petrovia, S. Lorenzo, Umago. Il punto estremo esplorato era posto a 12,6

53 "Relazione della Giunta provinciale sull'infezione filosserica in provincia e sulle misure di repressione attuate nell'anno 1881-82", Atti della Dieta provinciale istriana, Parenzo, 19 agosto 1882.

54 "Protocollo dell'ottava seduta della Commissione provinciale pei provvedimenti contro la fillossera", Atti della Dieta provinciale istriana, Trieste, 24 luglio 1882; "Relazione sui lavori intrapresi dal luglio 1882 al marzo 1883 nella regione infetta dalla filossera in Istria”, Atti della Dieta provinciale istriana, Gorizia, 23 aprile 1883, XLI-XLII.

55 "Protocollo della IX seduta della Commissione provinciale pei provvedimenti contro la filossera", Atti della Dieta provinciale istriana, Trieste, 13 maggio 1883, XLV. 
km di distanza dal centro d'infezione di Pirano. Nei distretti politici di Capodistria, Parenzo e Pisino vennero istituite delle Commissioni locali per i provvedimenti contro la fillossera, con il compito di assistere le autorità politiche distrettuali. Tali provvedimenti permisero di scoprire un'ulteriore e preoccupante diffusione sia nei comuni già infettati, Pirano, Isola e Salvore, che in quelli di Corte d'Isola ed Umago. Il male allora occupava una superficie di $62 \mathrm{kmq}$, e le macchie periferiche ebbero origine da trasporti artificiali ed inopportuni, sia attraverso le mani degli operai, che con il trasporto, più frequente, di canne già usate quali pali da sostegno. ${ }^{56}$

Per conoscere più a fondo la diffusione della fillossera, il Ministero dell'agricoltura ordinò fin dal 1883 una serie di studi atti ad indagare tutte quelle circostanti utili alla lotta nelle regioni istriane infette, attinenti sia alla viticoltura che alla fillossera. Si praticarono le osservazioni ampelografiche e le analisi dei vitigni indigeni, utili a definire le varietà di maggior pregio e di conseguenza meritevoli alla conservazione, e per precisare, assieme alle immagini ed agli acquarelli, la sinonimia con gli eventuali vitigni coltivati altrove sotto differente nome. Risultava inoltre necessario stabilire quali di esse rendevano più efficace l'azione degli insetticidi. Vennero esaminate le seguenti varietà: Rifosco, Rifoscara, Terrano, Rossara, Negra tenera, Sanzene, Verdaccia, Biancara, Isolana, Bontempa, Pagadebita, Malvasia e Moscato. Si eseguirono pure le analisi fisiche e chimiche dei terreni di origine eocenica - marne e tasselli d'Isola, e dei terreni alluvionali della Valle di Sicciole. Ciò per procedere con razionalità alle concimazioni supplementari ai trattamenti colturali. Si analizzarono pure i fertilizzanti impiegati nelle concimazioni sperimentali, gli insetticidi in uso e la conoscenza del modus vivendi ed i processi di moltiplicazione e di propagazione dell'insetto nelle condizioni istriane. ${ }^{57}$

Le autorità provinciali continuarono ad insistere, inutilmente, presso il Ministero dell'agricoltura affinché si proceda alla coltivazione dei vitigni americani in via sperimentale. Nulla però vietava ai possidenti di procedere per via privata a tali esperimenti. Nel territorio infetto era però impossibile trovare un vivaio all'uopo, vista l'esorbitanza dei prezzi e la qualità dei terreni. Sorsero inoltre dei dubbi circa la valenza normativa di tali risultati per le altre parti dell'Istria. Era inoltre da preventivare il fatto che molti viti-

56 "L'infezione fillosserica in Istria nel 1884. Relazione di G. Bolle", Atti della Dieta provinciale istriana, Parenzo 1885, allegato n. 22, LXVII-LXXX.

57 Idem, LXXVI-LXXX. 
coltori, per provvedere alle conseguenze della fillossera avrebbero tentato d'introdurre abusivamente la coltivazione delle viti americane, favorendo l'eventuale diffusione di nuovi focolai infetti. Perciò si giunse a proporre un compromesso: si rinunciò all’idea di erigere dei vivai di viti americane nei territori fillosserati di Pirano ed Isola, per procedere alla distribuzione di viti americane ai possidenti residenti in detti comuni. In tal caso doveva trattarsi di viti già provate e resistenti quindi al male, da scegliersi preferibilmente tra quelle adatte al portinnesto: York-Madeira, Riparia, Rupestris, ecc, ritirabili nei luoghi sicuri ed immuni alla fillossera, preferibilmente a Berlino, Marburg e in Carinzia. Si proibì però l'esportazione di queste talee all'infuori di detti comuni. Inoltre, per proibirne ulteriormente l'esportazione, si propose l'istituzione di due vivai provinciali, di cui uno presso la Stazione eno-pomologica di Parenzo, e l'altro nell'orto succursale di Pisino. ${ }^{58}$

Lispezione fillosserica del 1885 interessò i territori comunali già infetti, e quelli vicini di Castelvenere, Momiano, Buie, Petrovia e S. Lorenzo e non diede ulteriori preoccupazioni. Complessivamente, l'area totale istriana infetta ammontava allora a 83,7 ettari, con un aumento complessivo di 24 ettari. In un solo anno l'area istriana infetta era aumentata del 28,7\% .

L'anno seguente si ebbe un aumento di 44,7 ettari, pari al 35\% circa. Larea infetta si era estesa ai comuni locali di Paugnano (Carcauze), Buie (Merischie e Zuppiga) ed Umago (Bassania). ${ }^{60}$

Nel 1886, le autorità viennesi concessero finalmente l'introduzione delle viti americane nei territori infetti. Vennero allora distribuite 5000 talee. Tale processo non fu immune dai problemi, essendo le talee molto sottili ed avendo difficoltà ad emettere radici quelle di Riparia. La loro vegetazione non fu molto appariscente e si notavano casi di clorosi. Gli innesti di refosco sulla Riparia allevata da seme praticati nel carso di Salvore erano pure insoddisfacenti. Le autorità provinciali s'assunsero un terzo delle spese d'indennizzo mentre, basandosi sulla Legge dell'Impero del 3 giugno 1885, lo stato se ne caricò pure uno. In tal modo, si pose la proprietà terriera in una posizione assai più favorevole rispetto all'applicazione della precedente

\footnotetext{
58 "Protocollo della XII seduta della Commissione provinciale pei provvedimenti contro la filossera", Atti della Dieta provinciale istriana, Trieste, 8 febbraio 1886, LX-LXI.

59 Bolle, „L'infezione filosserica in Istria nel 1885“, Atti della Dieta provinciale istriana, Gorizia, 17 febbraio 1886, LXII e LXV.

60 Idem, „L'infezione fillosserica in Istria nel 1886“, LII-LIII.
} 
legge in merito del 1875 . Vennero inoltre istituite le Commissioni fillosseriche locali nei comuni di Pirano, Isola, Capodistria, Paugnano, Umago, Buie, Bogliuno e Pisino. Si propose pure l’istituzione di appositi esploratori fillosserici appresso dette commissioni, nominati dalla Luogotenenza su proposta del dirigente fillosserico provinciale. ${ }^{61}$

Progressivamente, la fillossera toccò i territori di Capodistria e Verteneglio (1877), Lussingrande (1888), Muggia e Dolina (1889), Lussinpiccolo ed Ossero (1890), Ponte e Verbenico (1891), Bellai e S. Giovanni sull'isola di Cherso, Veglia e Grisignana (1892), Parenzo (1893), Visinada e Cittanova (1894), Pisino e Montona (1895). ${ }^{62}$ Nei primi anni del XX secolo nuovi focolai d'infezione vennero scoperti nell'Istria meridionale, nel distretto di Volosco e a Sansego. ${ }^{63}$

Risultarono distrutti 1200 ettari di vigneti, ossia 10 milioni di viti, con una perdita annuale del prodotto pari a circa un milione di fiorini. Ciò indusse la Giunta provinciale a rivolgersi ancora una volta alla capitale per accordare all'Istituto agrario provinciale il permesso d'importare dalla Francia una limitata quantità di talee di viti americane adatte ai terreni calcarei cosiddetti "biancuzzi”, visto che la Riparia, la Solonis e la Rupestris non risultarono adatte ad essi, causa la clorosi che ne ostacolava l'innesto. Si trattava di varietà derivate dall'incrocio della Vitis Berlandieri con la Vitis vinifera, indispensabili alla ricostituzione dei vigneti nei territori fillosserati di Muggia, Capodistria, Isola, Pirano, Buie, Visinada, Pisino, Albona e Veglia. ${ }^{64}$

La risposta ministeriale fu negativa. Allo stesso scopo, si promosse l'erezione ad Isola di una vigna sperimentale tesa a studiare le varietà di viti americane adatte all'occorrenza. Agli inizi degli anni Novanta, si proseguì con l'impianto di viti americane nei territori infetti. L'impianto di viti americane progrediva a Pirano, Isola, Umago e Capodistria. Inoltre, a Pirano, Isola, Capodistria, Lussingrande, Ossero, nel Goriziano e nel territorio di Trieste erano attivi i vivai sperimentali. ${ }^{65}$ Ancora, su iniziativa del Consiglio

61 "Protocollo della II seduta della Commissione provinciale istriana pei provvedimenti contro la fillossera tenutasi a Parenzo addì 2 Aprile 1887", Atti della Dieta provinciale istriana, Parenzo 1887, XLII.

62 "Relazione sull'attività del Consiglio agrario provinciale dell'Istria", Atti della Dieta provinciale istriana, Parenzo 1896, 28; "Protocollo della I seduta della Commissione provinciale dell'Istria pei provvedimenti contro la fillossera, tenutasi a Parenzo addì 12 ottobre 1892" (in seguito: "Protocollo della I seduta 1892"), Atti della Dieta provinciale istriana, Parenzo 1892, LXXXVII.

63 "Relazione sull'attività del Consiglio agrario provinciale nel 1901", Atti della Dieta provinciale istriana, Parenzo 1902, 12.

64 Atti della Dieta provinciale istriana, Parenzo 1892, allegato n. 27, XXXVIII-XXXIX.

65 "Protocollo della I seduta 1892", XXXVI-XXXVIII. 
agrario provinciale, si eressero dei filari modello d'innesti su viti americane a Pirano, Capodistria e Buie. Si diffuse il mercato nero, in cui la qualità e la resistenza delle viti non erano provate. Molte delle viti sperimentate ed impiantate risultarono essere inadatte, addirittura intaccate dalla fillossera ed in deperimento. Tale era il caso degli impianti di Othello, Noah, Jacquez e Vialla su di un fondo fillosserato nella Valle Grande di Sicciole. Si presentavano resistenti invece il York-Madeira, la Riparia, la Rupestris ed il Solonis. Alcune esperienze del 1893 dimostrano come singole qualità piantate in precedenza su di una particella in parte marnosa ed in parte argillosa, presentavano dei risultati alquanto diversi. Nella parte marnosa esse risultarono essere clorotiche e di scarsa vegetazione. Al contrario, sul versante argilloso esse ebbero una vegetazione molto rigogliosa e presentavano pure dei tralci molto lunghi. Anche la siccità mise il suo zampino. In conseguenza di essa, gli innesti eseguiti nel 1893 non riuscirono del tutto. ${ }^{66}$

Le autorità centrali intervennero ancora una volta a favore dei territori fillosserati, promuovendo la Legge 28 maggio 1892, relativa a concessioni di favori e sussidi a sollievo dei danni provocati dalla fillossera, sotto forma di anticipazioni statali, vincolati ad un'anticipazione uguale dai fondi provinciali. Il comune di Isola fu tra i primi fra quelli istriani colpiti, a farne richiesta, ottenendo il parere favorevole sia della Giunta provinciale, che degli esperti. ${ }^{67}$

Verso la fine del secolo, la giunta provinciale constatava amaramente l'inerzia e la leggerezza con cui in tutti questi anni si era affrontato il problema. Le commissioni fillosseriche locali non si erano sempre dimostrate all'altezza dei compiti affidati. Molte di esse non si riunirono mai, altre affrontarono l'emergenza con leggerezza. Spesso a membri di tali organismi venivano eletti dei soggetti inadatti ed incapaci a riconoscere il parassita. Molti periti locali erano pure impreparati. Talvolta si dimostrava carente anche l'attività della commissione provinciale. Tale situazione, a cui andava aggiunta la prolungata mancata convocazione della commissione centrale con sede a Vienna, generò spesso il malcontento nelle file della Dieta provinciale.

66 Giuseppe Ursic, "Relazione sullo stato dell'infezione fillosserica nel Litorale alla fine dell'anno 1893, e sui provvedimenti adottati”, Atti della Dieta provinciale istriana, Parenzo 1894, LXXXVI.

67 "Relazione e proposte della Giunta provinciale sulla domanda del Comune d'Isola per un'anticipazione dal fondo provinciale, allo scopo della ricostituzione di parte dei vigneti distrutti dalla fillossera", Atti della Dieta provinciale istriana, Parenzo 1893, 1-2; "Parere tecnico e piani di coltura, di liquidazione e di controllo per l'attivazione del prestito al Comune d'Isola, pel reimpianto dei vigneti distrutti dalla fillossera”, Atti della Dieta provinciale istriana, Parenzo 1893, 3-13. 


\section{Le iniziative degli anni Ottanta}

Agli inizi degli anni Ottanta, parallelamente alla comparsa delle peronospora e della fillossera, si fecero sempre più insistenti le richieste d'ammodernamento agrario e le necessità di conoscere lo stato reale dell'economia agricola, con i suoi pregi e difetti, le necessità e le possibilità di sviluppo, ecc. Fu soprattutto la nascente Società agraria istriana a prendersi cura di ciò. E se forse qualche cosa s'era fatto, si notavano ancora dei grossi problemi, quali la mancanza di strade e vie di comunicazione, seppur migliorate pochi anni prima con l'inaugurazione della ferrovia istriana nel 1876 e della "Parenzana" nel 1902, che resero più vicini i mercati, e favorirono le comunicazioni tra le genti dell'interno e quelle della costa istriana.

La Monarchia asburgica s'impegnò pure alla cura dei boschi ed all'imboschimento della provincia istriana. Si tracciarono i confini, contrassegnati da pietre di demarcazione con sopra incisa la numerazione ordinaria. ${ }^{68}$ Nel 1882, ebbe inizio l'attività d'imboschimento, sovrintesa dall'apposita Commissione. ${ }^{69}$ Inizialmente, la sua attività interessò i distretti di Capodistria, Pisino e Volosco, per poi estendersi anche ad altre aree istroquarnerine. Dette iniziative, nonostante i modesti mezzi stanziati, furono portate avanti con successo, visti gli ingenti profitti ricavati dall'economia forestale. ${ }^{70}$

Nel 1889 ci fu la partecipazione all'Esposizione agricola forestale di Vienna (18 ottobre 1889 - settembre 1890). Si trattò di un'ottima occasione per trattare delle condizioni dell'economia agraria istriana, poco apprezzata e scarsamente conosciuta. In questa sede si poté anche constatare un'ulteriore peggioramento della situazione, visto che le cose volsero al peggio, dopo che l'economia agricola europea assunse un carattere prettamente industriale, che mal si amalgamava con i connotati tradizionali di quella peninsulare. ${ }^{71}$

Contemporaneamente, si generò nella penisola una coraggiosa sorta di offensiva a carattere promozionale ed una ricerca di nuovi mercati, dai tratti non sempre soddisfacenti, visto che ancora una volta vennero fuori i limiti

68 Ivan Milotić, "Povijest življenja na području Općine Oprtalj” / "La storia della vita nel Territorio del Comune di Portole", Oprtalj - Portole, a cura di Vladimir Lay et al., Portole 2009, 35.

69 “Per l'imboschimento della Provincia (A. Davanzo)", L'Istria agricola, n. 2, 15 dicembre 1907, 30-33.

70 Elio Apih, "Qualche notizia sull'attività di rimboschimento in Istria avanti la Prima guerra mondiale", ACRSR, VI, 1975-76, 231-241.

71 "Relazione generale alla Dieta provinciale del Margraviato d'Istria sulla gestione della Giunta provinciale dalla sessione dietale dall'anno 1889 in poi" (in seguito: "Relazione generale alla Dieta provinciale"), Atti della Dieta provinciale istriana, Parenzo 1890, 17. 
produttivi regionali nei vari settori. Nel 1884 l'Istituto agrario parentino portò in Francia trenta tipi di vino analizzato e proveniente da diversi vigneti istriani. L'esperienza fu drammaticamente crudele: soltanto quattro vini di Parenzo, Capodistria, Verteneglio e Dignano ebbero un giudizio positivo.

Va ricordato anche un altro ostacolo a cui i produttori istriani - e non solo loro - andarono incontro nella seconda metà del XIX secolo ed agli inizi di quello successivo: la liberalizzazione del mercato sia interno che internazionale, il che metteva fine alle loro certezze, dovuto allo smercio dei prodotti. In questo contesto, fu proprio la vitivinicoltura, in quanto settore produttivo maggiormente esposto, a subirne le conseguenze. Il che generò malcontenti e dissapori, soprattutto al momento in cui la Duplice monarchia iniziò ad importare vino dalla penisola italiana. Infatti, stando ad una clausola integrante, l'accordo di collaborazione economica fra essa ed il Regno sabaudo, l'Austria-Ungheria concesse l'importazione di vino dalla penisola appenninica, a cui i produttori istriani si opposero con energia. Così ad esempio, i vitivinicoltori istriani presenti alla prima esposizione e degustazione del vino regionale che si tenne a Pisino nel 1901 affermarono di poter vendere al massimo trenta ettolitri di vino circa, ossia una quantità pari a quella contemporaneamente importata da Bari nella Monarchia. ${ }^{72}$ Perciò essi proposero al governo centrale di introdurre delle misure doganali protezionistiche sul vino importato dalla penisola italica, a tutela del prodotto locale, sulla falsariga di quanto avveniva in altri stati europei. Ricorderemo l'esempio francese, che causerà la cosiddetta "guerra del vino" fra Italia e Francia nel 1887.

\section{L'allevamento animale}

La Dieta provinciale s'impegnò pure a favore dell'allevamento animale, proponendo delle iniziative in merito. Tra le prime proposte del governo provinciale, da rilevare quella dell'esclusione decennale da qualsiasi forma di tassazione dell'animalia grossa, a partire dal 1866.

Ci furono allora dei progressi, soprattutto nel piranese e nel buiese, dove ormai si andava verso una forma di allevamento di vacche lattifere ed in grado di migliorare sia lo stato dell'animalia che la condizione econo-

72 Pučki prijatelj. Ilustrovani poučno-gospodarski list (L'amico del popolo. Foglio educativo-economico illustrato), a. 2, Veglia, 10-VI-1901, n. 11, 170. Sull'esposizione vinicola di Pisino, si rimanda a Davor Šišović, "Prvi istarski javni pokus vina u Pazinu" ("Il primo assaggio pubblico del vino a Pisino"), Stoljéé vina 1901. 2001., 9-14. 
mica dei produttori. ${ }^{73}$ Complessivamente si ebbe nella penisola un progressivo aumento dei bovini e dei suini, che non portò a dei grossi vantaggi per l'economia agricola. Le capacità bovine continuarono ad essere generalmente usate alla sola forza lavorativa, e la loro produzione di escrementi rimaneva insufficiente alle necessità.

Alla IV mostra distrettuale bovina, svoltasi a Buie, le armente erano considerate superiori di merito rispetto ai tori, parecchi dei quali presentavano un'ossatura debole ed una statura deficiente. Successivamente, s'istituirono le stazioni di monta suina ed ovina. ${ }^{74}$

Si procedette inoltre all'acquisto di microscopi e degli altri utensili utili allo sviluppo della bachicoltura ed alla prevenzione delle malattie. Ciononostante, tale industria non progredì molto.

\section{Gli anni Novanta}

Le linee ferroviaria Trieste - Pola nel 1876 e Trieste - Buie - Parenzo nel 1902, contribuirono ad avvicinare maggiormente le piazze di mercato all'entroterra agricolo. Si acquisirono esperienze internazionali, partecipando con i prodotti provinciali alle fiere di mercato ed esposizioni varie. Si organizzarono le prime associazioni sindacali e di categoria, le cooperative, le cantine vinicole, e si diffusero i moderni oleifici. Si diffusero riviste e trattati specialistici, ed anche la stampa ebbe un ruolo importante nella diffusione delle cognizioni scientifiche agrarie. ${ }^{75}$

Nonostante questi positivi eventi, stentava ancora l'affermazione dei principali prodotti istriani sui mercati: il vino istriano per esempio, non era ancora in grado di contrastare gli avversari interni, ne tantomeno quelli esterni. Alle varie mostre enologiche sia nazionali che internazionali alle quali esso partecipava, i risultati erano molto scadenti. ${ }^{76}$

Nel 1892 la Giunta provinciale constatava i progressi agronomici ovviamente contrastati dalla crisi fillosserica in atto - frutto pure del lavoro scientifico portato avanti dall'Istituto agrario parentino. Proprio in conseguenza del ruolo che esso aveva assunto e delle nuove sfide che attendevano

73 "Relazione della Presidenza della Società agraria all'I. R. Ministero dell'agricoltura", La Provincia dell Istria, a. V, n. 5, 4 marzo 1871, 695.

74. "Relazione generale alla Dieta provinciale", 18.

75 Roberto Starec, "La trattatistica istriana dell'800 sulla viticultura", ACRSR, XXVIII, 1998, 599-609.

76 Denis Visintin, "La vite nella geologia, nella preistoria e nelle civiltà preromane. America, rinnovato vigore", Panorama, a. L, n. 19, Fiume, 15 ottobre 2001, 23; Vido Vivoda, "Vinogradarstvo i vinarstvo Istre na prijelazu iz 19. u 20. stoljeće" ("La vinicoltura e l'enologia dell'Istria a cavallo tra il XIX e il XX secolo"), Stoljeće vina 1901.-2001., 45. 
dietro l'angolo, la dirigenza provinciale si fece interprete delle richieste fatte dal direttore Carlo Hughues, circa il potenziamento dell'appoggio finanziario governativo. Per cui la Giunta provinciale appoggiò il richiesto aumento delle dotazioni statali, relativo alla manutenzione degli impianti sia a Parenzo che alla sezione di Pisino da 2 a 3000 fiorini, e lo stanziamento di un contributo di 800 fiorini a favore della Scuola pratica biennale, e di 500 fiorini a sostegno del corso di agronomia per i possidenti agricoli. Tutto sommato, si trattava di stanziare una somma complessiva pari a 4300 fiorini. Una cifra quindi modesta, rispetto alle reali esigenze. ${ }^{77}$

La situazione fu turbata dal rinnovo del Trattato commerciale fra Austria-Ungheria e Regno d'Italia, cosa mal digerita dalle rappresentanze istriane di categoria, rimaste al margine delle trattative, e poste di fronte al fatto compiuto. Entrando esso in vigore, nell'agosto del 1892, venne attuata pure la clausola relativa all'importazione di vini italiani ed alla loro commercializzazione nelle terre asburgiche. L'impressione che se ne ricavò era che la Duplice monarchia non si preoccupò affatto della tutela dei suoi vini. In conseguenza di ciò si ebbe una maggiorata concentrazione di vendita dei vini istriani sulla piazza triestina. Di conseguenza, si ridussero le potenzialità mercantili di consumo, e le nuove sfide, anche quelle relative alla liberalizzazione del mercato interno ed esterno, andavano vinte con il continuo miglioramento delle tecniche produttive, e di conseguenza del prodotto. Perciò, il supporto e l'attività dell'Istituto agrario parentino erano fondamentali. Con gli inizi degli anni Novanta, esso ampliò le sue ingerenze agli altri rami agricoli: cerealicoltura, olivicoltura, orticoltura, praticoltura, zootecnia, divenendo così un reale punto di riferimento per tutta l'Istria agricola, e non soltanto per le aree viticole. Si rese allora necessario un affronto comune ai tempi nuovi che si andavano affermando. Di conseguenza, fecero la loro comparsa le associazioni di categoria e sindacali, le cooperative agricole e, più in la, le cantine vinicole sociali.

\section{I disastri climatici di fine secolo}

Tutto ciò succedeva mentre altri pericoli oscuravano le annate produttive. A seguito di una serie di disastrosi eventi climatici di fine secolo, vennero danneggiate la vegetazione viticola e la fioritura dei frutteti. I gelsi vennero colpiti dalla ruggine. Le inclemenze iniziarono con il freddo inverno del

77 "Relazione generale della Giunta alla Dieta provinciale sulla gestione della chiusa dall'anno 1892 in poi”, Atti della Dieta provinciale istriana, Parenzo 1893, XXXIV-XXXV. 
1892, cui fece seguito una primavera piovosissima e con temperature incostanti. Nella zona degli altipiani, in collina e sul Quarnero si ebbero notevoli danni alle colture cerealicole, soprattutto al frumento ed ai prati. Nel mese di marzo, l'improvvisa neve ed il gelo distrussero gran parte dei piselli, dei legumi e degli ortaggi lungo la costa nord - occidentale, mentre la bora danneggiò gli alberi da frutto, e nell'area quarnerina anche gli ulivi. In generale, il biennio 1891-92 vide l'abbattersi di disastrose grandinate. La costa occidentale, da nord a sud, ed alcune aree interne, subirono delle notevoli grandinate, particolarmente i territori di Umago, Parenzo, Valle, Canfanaro, Dignano, Barbana, Parenzo, Capodistria, Isola, Pirano, Pedena, Sovignacco, Gallignana. Danni dovuti alle grandinate si ebbero pure nei territori di Albona, Laurana, Castua, Dobrigno ed Apriano. ${ }^{78}$

L'anno seguente fu caratterizzato dalla siccità che colpì quasi tutta la penisola, in modo particolare il parentino, seguita dal gelidissimo inverno, in cui le temperature giornaliere erano quasi sempre sotto lo zero. Le basse temperature perdurarono fino a primavera inoltrata, con gravi danni per le colture arative, particolarmente il mais nel polese e nel quarnerino e le viti, specie nell'albonese, per gli ortaggi, e per la coltura olearia. La vendemmia fu invece copiosa, date soprattutto le eccellenti condizioni climatiche che si ebbero nella fioritura di maggio. Nell'Istria centrale e lungo li versante nordoccidentale, essa fu guastata dalle grandinate. ${ }^{79}$

Particolarmente colpiti furono, negli anni 1894-95, i territori della bassa Istria e dei suoi versanti settentrionale, nordorientale ed occidentale. La siccità invernale ed estiva, intercalata da una piovosa e fredda primavera rovinarono la vegetazione viticola e la fioritura dei frutteti. Mentre la gelsicoltura fu colpita dalla ruggine. La grandine fece pure danni. ${ }^{80}$

Il lungo e piovosissimo inverno 1895 fu seguito da una primavera altrettanto piovosa e desolante, e da un'estate secca. Al contrario, l'anno seguente si ebbero un inverno ed una primavera eccezionalmente asciutti e un'estate incessantemente piovosa. L'annata fu decisamente svantaggiosa per i pascoli ed i foraggi, per cui si dovette procedere all'importazione dei foraggi dalla Carniola per alimentare gli animali. Le semine primaverili furono danneggiate dalla mancanza d'acqua, mentre le piogge estive

78 "Relazione sull'attività del Consiglio agrario provinciale dell'Istria nel periodo 1892-93", Atti della Dieta provinciale istriana, Parenzo 1894, 26-27.

79 Idem, 27.

80 Rino Cigui, “La crisi agricola degli anni 1894-95 ed i suoi riflessi sociali”, La Ricerca, 59, 2011, 17-19. 
causarono enormi perdite ai prodotti maturati. Anche le colture frutticole, olearie e viticole furono danneggiate dalla pioggia prima, e dai forti venti dopo. $^{81}$

Le piogge continuarono cruentemente nelle successive stagioni autunnale, invernale e primaverile del 1897. Seguì una siccitosa estate, una delle più asciutte degli ultimi anni, che si protrasse fino alla prima decade di settembre.

Lagricoltura presentava uno stato misero e desolante. Furono tristi innanzitutto le condizioni cerealicole rese ulteriormente difficili dalla ruggine del frumento. Le viti, indebolite dalla stagione piovosa dell'anno precedente, subirono una ritardata germogliatura primaverile, e gli aggravi dovuti alla peronospora, alla filossera, alle piogge ed ai freddi tardivi, la siccità estiva e le grandinate complicarono ancor di più lo stato delle cose. Anche gli altri raccolti ebbero delle rendite inferiori alla media. ${ }^{82}$

Più o meno simile fu l'andamento nel 1898. La siccità si fece sentire tra aprile e la metà di giugno. Seguì un brutto periodo piovoso, che si protrasse fino alla fine di settembre. Fallì quasi dappertutto il raccolto del mais primaverile, ed una sua seconda semina fu resa pure impossibile. Vennero danneggiate pure le patate, i legumi e gli altri ortaggi, i prati e le colture estive. Risultò discretamente riuscita la raccolta del frumento, della segale, dell'avena, dell'orzo, delle fave, e generalmente di tutte le semine autunnali ed invernali. Ciò favorì l'arresto del prezzo delle farine, in salita causa la generale carestia dei grani, che perdurava da due anni. Va sottolineato però che nella Monarchia asburgica di quegli anni si era puntato molto sullo sviluppo monoculturale del grano. La scelta appariva scontata proprio in conseguenza del crollo delle quotazioni granarie sul mercato mondiale. ${ }^{83}$ La mancanza d'acqua si fece sentire in molte parti, con grave danno anche per l'animalia, dato il prosciugamento di molti lacchi. Di conseguenza ci si dovette rivolgere ad altri territori per l'approvvigionamento. La gelsicoltura e la frutticoltura diedero al contrario un raccolto molto scarno. La vendemmia fu danneggiata dalla fillossera, dalla peronospora, dall'oidio e delle grandinate. Queste ultime danneggia-

81 "Relazione sull'attività del Consiglio agrario provinciale nell'anno 1896 con cenni riassuntivi sull'operato nel sessennio 1891-96 e sull'impiego delle dotazioni erariali fino al 1895", Atti della Dieta provinciale istriana, Parenzo 1896, 23.

82 "Relazione sull'attività del Consiglio agrario provinciale dell'Istria nel periodo 1897-98", Atti della Dieta provinciale istriana, Parenzo 1899, 27-28.

83 Battisti, "Di crisi in crisi", 30. 
rono pure le olive, soprattutto nei distretti di Cittanova, Parenzo, Valle, Capodistria e Pirano. ${ }^{84}$

Anche nelle aree vicine, vedi ad esempio la Pianura padana, la crisi agraria favorì l'aumento del prezzo del grano e pure della canapa. Di conseguenza, si ebbe un deciso cambiamento di rotta economica, con la riduzione dei prati, lo sradicamento delle colture storiche ritenute improduttive e di quelle legnose, l'investimento di capitali nell'allevamento animale, l'avvento della meccanizzazione e dei lavoratori salariati. Finiva l'epoca delle famiglie patriarcali ed iniziava quella dell'industrializzazione. ${ }^{85}$ Cosa che invece la penisola istriana non fu in grado di fare.

\section{Conclusione}

Il raggiunto grado di progresso peninsulare venne messo in mostra alla Prima esposizione provinciale istriana, che si tenne a Capodistria nel 1910 dal primo maggio alla fine di settembre. ${ }^{86}$ Lesposizione presentava tutto l'utile allora in circolazione ad uso agricolo: fertilizzanti chimici, macchine agricole, materiale didattico, prodotti del suolo, utensili da lavoro, botti e contenitori da olio e da vino, oleifici meccanici.

Si trattò di un confronto teorico-scientifico e pratico che vedeva da un lato esposte le migliori esperienze provinciali, e dall'altro quanto di meglio potevano offrire le vicine regioni austro-ungariche e le aree europee contermini, in cui l'agricoltura provinciale, notevolmente deficitaria, indicava allora i limiti estremi raggiunti dai suoi processi evolutivi.

La penisola non era in grado di supportare il decollo agricolo, anche se buona parte della popolazione era entrata nel giro dell'economia di mercato. Tuttavia, si entrava lentamente in un'epoca in cui si verificarono notevoli cambiamenti sociali, politici ed economici, ed in cui l'economia agricola tradizionale s'avviava, seppur lentamente, verso il tramonto.

Seppur la situazione agricola non era brillante, la situazione d'inizio secolo era alquanto diversa rispetto al periodo precedente. La padronanza agraria, liberata dagli obblighi d'acquisto del suolo esonerato, poteva indirizzare gli sforzi finanziari verso ulteriori traguardi di rinnovamento. I

84 "Relazione sull'attività del Consiglio agrario provinciale dell'Istria nel periodo 1897-98", 28.

85 Gianluigi Della Valentina, "Padroni, imprenditori, salariati. Modelli capitalistici padani”, Storia dell'agricoltura italiana, II. Uomini e classi, a cura di Piero Bevilacqua, Venezia 1990, 193-195.

86 Catalogo generale della Prima esposizione provinciale istriana, Capodistria 1910; "Gli agricoltori istriani premiati all'esposizione di Capodistria“, L'Istria agricola, a. 3, n. 20, 8 ottobre 1910, 494-497; Prima Esposizione Provinciale Istriana - 100 anni, a cura di Dean Krmac, Capodistria 2010. 
vigneti fillosserati erano in gran parte recuperati, salvo qualche focolaio che ancora si presentava nella penisola, avanzava il sapere agrario e fecero la loro comparsa le prime associazioni cooperativistiche e le Cantine vinicole pubbliche.

\section{Riassunto}

Nel corso della seconda metà del XIX secolo, il settore agrario istriano fu interessato da tutta una serie di trasformazioni. Da un lato ciò era dovuto alla politica modernizzatrice che interessò tutta la Monarchia asburgica, dall'altro ci fu la spinta dal basso, ossia dalle autorità provinciali. Nonostante ciò, questo processo, già tardivo rispetto ai territori europei occidentali, fu lento. Le cause andavano ricercate negli ostacoli talvolta posti dalle autorità centrali, nell'inadeguatezza di talune amministrazioni locali, nel disinteresse della padronanza, nelle crisi che colpirono il settore, nel diffuso analfabetismo e nella mancanza di scuole ed associazioni diffondenti il sapere agrario.

Il settore fu interessato da gravi crisi. Particolarmente duro fu il periodo 1851-63, complici la comparsa dell'oidio, dell'atrofia dei bachi da seta, le dure inclemenze meteorologiche e le carestie. Anni di crisi si ebbero anche più avanti, con la carestia di fine anni Settanta, la diffusione della peronospora e della fillossera, nonché nel decennio di fine secolo.

Con l'abolizione del feudalesimo, che in Istria ebbe una fase duratura e prolungata, iniziò una fase liberale e d'ammodernamento dell'agro istriano, caratterizzata dalla progressiva liberalizzazione del mercato, dall'avvento del capitale nelle campagne, delle banche agrarie, delle associazioni di categoria e sindacali, della Scuola e dell'Istituto agrario parentini. Si trasformarono i sistemi colturali e si diffusero gli aratri metallici. La comparsa della solforazione, dei concimi, dell'innesto, del portinnesto, della selezione e della potatura delle viti segnò la fine dell'agricoltura tradizionale e l'avvento di quella nuova caratterizzata dall'intervento scientifico. Tuttavia, ancora agli inizi del XX secolo, l'agro peninsulare mostrava evidenti segni di debolezza.

\section{Istarska poljoprivreda u drugoj polovici XIX. stoljeća}

\section{Sažetak}

Od sredine XIX. stoljeća odvija se niz promjena u istarskom poljoprivrednom sektoru. $S$ jedne su strane te promjene bile posljedicom politike modernizacije koja se provodila u cijeloj Habsburškoj Monarhiji, a s druge su bile rezultat poticaja koje su poduzimale provincijske vlasti. Usprkos tome, taj se proces, ionako već u zakašnjenju u odnosu na razvoj u zapadnoeuropskim područjima, sporo odvijao. Tomu razloge valja potražiti u preprekama koje su katkad postavljale središnje vlasti, neprilagođenosti nekih lokalnih uprava, nezainteresiranosti posjednika, krizama koje su pogodile sektor, rasprostranjenoj nepismenosti te nedostatku škola i udruga koje bi širile znanje o poljoprivredi. 
Poljoprivredni su sektor pogodile duboke krize. Posebno je teško bilo između 1851. i 1863. zbog pojave pepelnice, atrofije dudova svilca, surovih meteoroloških prilika i velike oskudice. I kasnije je bilo kriznih godina, s nestašicama krajem sedamdesetih godina, širenjem peronospore i filoksere te u zadnjem desetljeću XIX. stoljeća.

Ukidanjem feudalizma, koji je u Istri proživio svoju produženu fazu, započelo je liberalno i modernizacijsko razdoblje istarskoga agrara, koje karakteriziraju progresivna liberalizacija tržišta, ulazak kapitala u poljoprivredu, agrarne banke, sindikalne i trgovačke udruge te djelovanje porečke poljoprivredne škole i instituta. Mijenjaju se sustavi usjeva i širi uporaba metalnoga pluga. Pojave sumporizacije, gnojidbe, kalemljenja, kalemljenja korijena, selekcije i orezivanja loze označile su kraj tradicionalne i pojavu nove poljoprivrede, obilježene znanstvenim intervencijama. Međutim, početkom XX. stoljeća istarska je poljoprivreda još uvijek pokazivala jasne znakove slabosti.

\section{Istrian agriculture in the second half of the $19^{\text {th }}$ century}

\section{Summary}

From the mid-19 $19^{\text {th }}$ century, many changes have occurred in Istrian agriculture. On the one hand, they were a consequence of the modernisation policy which was enforced throughout the Austrian Empire; on the other hand they were the result of enticements undertaken by provincial government. However, this process, already behind the more developed western areas, was very slow. The reasons for this can be found in the obstacles placed by the central government, ineptitude of some local authorities, land owners' indifference, and the crises which have hit the sector, the widespread illiteracy and lack of schools and associations which would spread the knowledge of agriculture.

Agriculture was hit by big crises. It was especially difficult between 1851 and 1863 because of powdery mildew, silkworm atrophy, bad weather and destitution. There were bad years later on as well, like privation in the late 70s, peronospora and phylloxera spreading during the last decade of the $19^{\text {th }}$ century.

Suspension of feudalism, which lasted longer in Istria, was followed by liberal and modernising period of Istrian agriculture which is characterised by progressive liberalisation of market, influx of capital in the agriculture, agrarian banks, syndicates and trade associations, as well as the activity of agricultural school and institute in Poreč. The system of crops changed, and the use of metal plough started spreading. The implementation of sulfidation, fertilization, grafting, root grafting, selection and vine pruning marked the end of traditional and arrival of new type of agriculture, marked by scientific input. Nevertheless, the Istrian agriculture still showed clear signs of weaknesses at the beginning of the $20^{\text {th }}$ century. 